\title{
Digitally-Assisted RF-Analog Self Interference Cancellation for Wideband Full-Duplex Radios
}

\author{
Kimberley Brynn King
}

\author{
A thesis \\ presented to the University of Waterloo \\ in fulfillment of the \\ thesis requirement for the degree of \\ Master of Applied Science \\ in \\ Electrical and Computer Engineering
}

Waterloo, Ontario, Canada, 2016

(c) Kimberley Brynn King 2016 
I hereby declare that I am the sole author of this thesis. This is a true copy of the thesis, including any required final revisions, as accepted by my examiners.

I understand that my thesis may be made electronically available to the public.

Kimberley Brynn King 


\begin{abstract}
The ever-increasing demand for more data from users is pushing the development of alternative wireless technologies to improve upon network capacity. Full-Duplex radios provide an exciting opportunity to theoretically double the available spectral efficiency of wireless networks by simultaneously transmitting and receiving signals in the same frequency band.

The main challenge that is presented in the implementation of a full-duplex radio is the high power transmitter leaking to the sensitive receiver chain and masking the desired receive signal to be decoded. This transmitter leakage is referred to as self interference and it is required that this self interference signal be cancelled below the receiver noise floor to achieve the full benefits of a full-duplex radio. Cancellation of the self interference signal is realized through several techniques, categorized as passive suppression, digital cancellation, and analog cancellation. These methods all have their challenges in achieving the full amount of cancellation necessary and therefore all three techniques are typically employed in the system.

In this thesis, a novel digitally assisted radio frequency ( $R F)$ analog self interference canceller is proposed to suppress the self interference signal before the receiver chain for wide modulation bandwidth signals. This canceller augments minimum complexity RFanalog interference cancellation hardware that uses an $\mathrm{RF}$ vector multiplier in combination with a flexible digital rational function finite impulse response filter. The simple topology reduces the number of impairments added to the system through the analog components and identifies the parameters of the proposed filter in a deterministic and single iteration algorithm.

The hardware proof-of-concept prototype is built using off-the-shelf RF-analog components and demonstrates excellent cancellation performance. Using four TX test signals with modulation bandwidths of $20 \mathrm{MHz}, 40 \mathrm{MHz}, 80 \mathrm{MHz}$, and $120 \mathrm{MHz}$, the self interference canceller achieves a minimum of $50 \mathrm{~dB}, 47 \mathrm{~dB}, 42 \mathrm{~dB}$, and $40 \mathrm{~dB}$ of cancellation respectively.

This thesis reviews the previously proposed self interference cancellation topologies, system non-idealities that provide challenges for full-duplex implementation, and the realization of the proposed RF-analog self interference canceller.
\end{abstract}




\section{Acknowledgements}

I would like to thank my supervisor, Dr. Slim Boumaiza, for giving me guidance, advice, and confidence throughout my Masters process. He has taught me how to properly conduct research, approach problems and allowed me to pursue a topic that suited my interests. The entire Emerging Radio Systems Group (EmRG) consists of intelligent, kind and helpful members who I have also learned a lot from and enjoyed working with.

I would also like to acknowledge my incredible family and friends who have offered continuous love and support throughout this lengthy Masters process.

Finally, I would like to dedicate this thesis to my husband, Michael, for his love, patience, and calming influence while pushing me to achieve my goals. 


\section{Table of Contents}

List of Tables vii

List of Figures viii

1 Introduction $\quad 1$

1.1 Motivation For Full-Duplex . . . . . . . . . . . . . . . . 1

1.2 Problem Statement . . . . . . . . . . . . . . . . . 2

1.3 Thesis Organization . . . . . . . . . . . . . . . . 2

2 Theory and Background $\quad 4$

2.1 Background For Full-Duplex . . . . . . . . . . . . . . . . 4

2.2 Full-Duplex Applications . . . . . . . . . . . . . . . . . 5

2.3 Self Interference Cancellation . . . . . . . . . . . . . . . . . 6

2.3.1 Passive Suppression . . . . . . . . . . . . . . . 7

2.3.2 Digital Cancellation . . . . . . . . . . . . . . 9 9

2.3.3 Analog Cancellation ................. . 10

2.4 System Nonidealities . . . . . . . . . . . . . . . . . . 15

2.4.1 ADC Quantization Noise . . . . . . . . . . . . 15

2.4.2 Linearity of the Transmitter, Canceller, and Receiver . . . . . . . 17

2.4 .3 Receiver Noise Floor . . . . . . . . . . . . . . . . 18

2.4.4 Oscillator Phase Noise . . . . . . . . . . . . . . . . . 19 
2.4 .5 Other Nonidealities . . . . . . . . . . . . . . . . . 20

2.5 Summary . . . . . . . . . . . . . . . . . . . . . . 21

3 Proposed Self-Interference Cancellation Approach: Theory and Design 22

3.1 Proposed Digitally-Assisted RF-Analog SI Cancellation Approach . . . . . 22

3.2 The Proposed Parameter Identification Algorithm . . . . . . . . . . . . . . 27

3.3 Canceller Implementation ． . . . . . . . . . . . . . . . . . . . . 28

4 RF Canceller Measurement Results $\quad 31$

4.1 Measurement Setup . . . . . . . . . . . . . . . . . . . . . . . . 31

4.1.1 Calibration of Measurement Setup _. . . . . . . . . . . 33

4.1 .2 CW Signal Cancellation . . . . . . . . . . . . . . . . . 34

4.1 .3 TX Test Signals . . . . . . . . . . . . . . . . . . . 35

4.1.4 Control Signal For Training . . . . . . . . . . . . . . . . 35

4.2 Measurement Results . . . . . . . . . . . . . . . . . . . . . . 36

4.3 System Analysis . . . . . . . . . . . . . . . . . . . . . . . 40

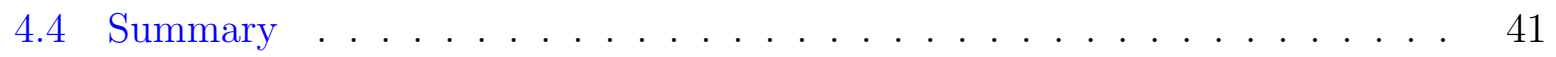

5 Conclusions 43

5.1 Future Works . . . . . . . . . . . . . . . . . . . . . . . 44

$\begin{array}{lr}\text { References } & 45\end{array}$ 


\section{List of Tables}

3.1 Active Component Parameters for the Proposed Canceller Experimental Setup 30

3.2 Passive Component Parameters for the Proposed Canceller Experimental Setup . . . . . . . . . . . . . . . . . 30

4.1 Summary of the Proposed SI Canceller Measurement Results . . . . . . . . 41

4.2 Performance Comparisons of Other RF-Analog SI Cancellers . . . . . . . . 42 


\section{List of Figures}

2.1 Spectrum and Time Utilization of FD Radios Compared to TDD and FDD [1] 5

2.2 SI Cancellation Mechanisms Within a FD Radio [1] . . . . . . . . . . . 6

2.3 Passive Suppression Through Multiple Antennas [2] . . . . . . . . . . . . 8

2.4 Transmit and Receive Operation of an Electrical Balance Duplexer [1] . . . 9

2.5 RF-Analog Cancellation Through Auxiliary TX Chain . . . . . . . . . . 11

2.6 RF-Analog Cancellation Through Magnitude and Phase Adjustment . . . . 12

2.7 Implementation of General Magnitude and Phase Adjustment Cancellation [3] 13

2.8 Implementation of Wideband RF-Analog Cancellation Through Multiple Taps of Variable Attenuators and Delay Lines [4] . . . . . . . . . . . . . . 14

2.9 Implementation of Wideband RF-Analog Cancellation Through Multiple Taps of Delay Lines and Vector Multipliers [5] . . . . . . . . . . . . 15

2.10 General FD Transceiver Topology For Impairment Analysis . . . . . . . . . 16

3.1 Block Diagram of the Proposed Digitally-Assisted RF-Analog Self Interference Cancellation . . . . . . . . . . . . . . . . 25

3.2 Block Diagram of an Ideal Vector Multiplier . . . . . . . . . . . . . . . . 26

3.3 Baseband Equivalent Behavioural Model of the Full-Duplex Radio Front-End 26

3.4 Experimental Setup of FD Radio Front-End Using Off-The-Shelf Components 29

4.1 FD Radio Equipment Setup and Connection . . . . . . . . . . . . . . 32

4.2 RF-Analog SI Cancellation of CW Signal with Constant Voltage Control Signal . . . . . . . . . . . . . . . . . . . . . 34 
4.3 RF-Analog SI Cancellation of $20 \mathrm{MHz}$ Modulation Bandwidth Signal . . . 37

4.4 RF-Analog SI Cancellation of $40 \mathrm{MHz}$ Modulation Bandwidth Signal . . . 38

4.5 RF-Analog SI Cancellation of $80 \mathrm{MHz}$ Modulation Bandwidth Signal . . . 38

4.6 RF-Analog SI Cancellation of 120 MHz Modulation Bandwidth Signal . . . 39

4.7 Number of ADC Bits Lost Due to the SI Signal and Require IIP3 Value of the LNA vs. the Received Signal Power . . . . . . . . . . . . . . . 41 


\section{Chapter 1}

\section{Introduction}

\section{$1.1 \quad$ Motivation For Full-Duplex}

Modern wireless communications usage has increased significantly in the past decade. With the introduction of smartphones and other connected devices, there has been a shift from voice-centric protocols to data-centric protocols and people are constantly looking for higher data rates and more bandwidth to support their demand for information. The licensed spectrum is becoming congested and we are fast approaching the theoretical capacity limit with the resources available today. A fundamental to radio design states that a transmitter and receiver of a node cannot operate simultaneously in the same frequency band. Therefore, current radios are designed in a half-duplex topology where the transmitter and receiver operate in different frequency bands called frequency domain duplexing (FDD) or at different times called time domain duplexing (TDD). With this architecture, it is not easy to support the growing population and growing demand.

To breakthrough this limit, in-band full-duplex (FD) techniques have been proposed and are being heavily researched. This change has the potential to double the spectral efficiency by simultaneously transmitting and receiving information in the same frequency band. It also provides more flexibility with the dynamic communication networks prevalent today. Creating a FD radio would involve some hardware changes to create a more cohesive and aware transmitter and receiver as well as certain wireless protocol changes that would utilize the extra bandwidth to its fullest extent. A shift from macro-cell base stations to higher density pico-cell or femto-cell base stations in the future, will also make FD radios more attractive. With this, the transmit power and the attenuation of the signal of interest will be slightly smaller, relaxing the components in the transmitter and receiver [6]. 


\subsection{Problem Statement}

With this growing interest in full-duplex, many people are researching implementation techniques to create reliable and flexible radios. However, there are many challenges preventing its immediate realization. The main issue stems from the transmitter signal leaking into the receiver chain, called the self interference (SI) signal. The outgoing signal is generally orders of magnitude larger than the incoming signal. This creates a very powerful SI signal that can completely drown out the signal of interest. Wired networks have already long implemented FD techniques with the separation of the two signal achieved over two wires. Wireless networks do not have such a complete solution for this separation so the SI signal remains large in the receiver. Active cancellation of this SI signal is required and various methods for cancelling have been proposed. Fortunately, the transmit (TX) signal is completely known ahead of time so it can be used to estimate the SI signal and create the necessary cancellation signal to be combined in the receiver chain.

Designing this entirely new system also involves many system-level tradeoffs and nonidealities that need to be properly optimized for a complete and practical solution. Adequately decoding the received signal of interest involves cancelling the self interference signal to a level below the receiver noise floor while preserving the necessary signal-to-noise ratio (SNR).

\subsection{Thesis Organization}

This thesis aims at developing and building a simple canceller to provide enough cancellation before the RX path so that the signal of interest can be accurately decoded. During this process, a full transmitter and canceller are built to measure SI cancellation before the RX chain.

The thesis is organized in the following manner to fully explore the implementation of a new SI cancellation method. Chapter 2 reviews applications for FD radios and where they provide the most benefit, categorizes the possible SI cancellation topologies, and reviews the previously proposed cancellation techniques in literature. This chapter also analyzes challenges that occur in the implementation of FD radios and what system-level decisions are necessary to achieve good performance.

A novel SI canceller is proposed in Chapter 3 that aims at suppressing the SI signal with simplified hardware for wide modulation bandwidth signals. The cancellation signal is developed using a single tap RF-analog topology and controlled through the use of a 
digital filter. The identification process for the parameters of the filter is developed in this chapter along with the hardware implementation of the system.

Chapter 4 presents the measurement setup for the initial prototype of the proposed FD radio and the results achieved by this RF-analog SI canceller over four TX test signals. It also analyzes the system nonidealities and compares its cancellation ability to other current designs.

Finally, Chapter 5 concludes this thesis with a summary of the benefits of the proposed solution and a discussion on the future work needed to provide a more complete solution. 


\section{Chapter 2}

\section{Theory and Background}

In this chapter the theory and background of full-duplex radio implementation is discussed and applications where FD radios pose an advantage over HD techniques is presented. Although the implementation of an analog canceller is the focus of this thesis, other common topologies are presented to show the progression of FD radio implementation. As well, system-level nonidealities and tradeoffs give context to the importance of the proposed cancellation technique.

\subsection{Background For Full-Duplex}

Modern wireless communication networks are growing at an enormous rate. People are trending towards using their mobile phones for more data-based services such as highdefinition (HD) video, which demands an increasingly large amount of data at a very fast data rate, all with the expectation of a reliable network. This continues to push the performance of wireless communications further. It has become very important to increase the spectral efficiency of these networks to ease the congestion by creating multi-mode and multi-band hardware for mobile phones and base-stations.

Conventional transceivers operate in a HD mode, transmitting and receiving in different time slots through TDD or in different radio frequency (RF) bands through FDD. While most current wireless networks are HD, FD communication technology has drawn significant attention recently. This is mainly due to its potential to theoretically double the spectral efficiency in the physical layer compared to its HD counterpart by allowing simultaneous transmission and reception of signals at the same frequency band. 

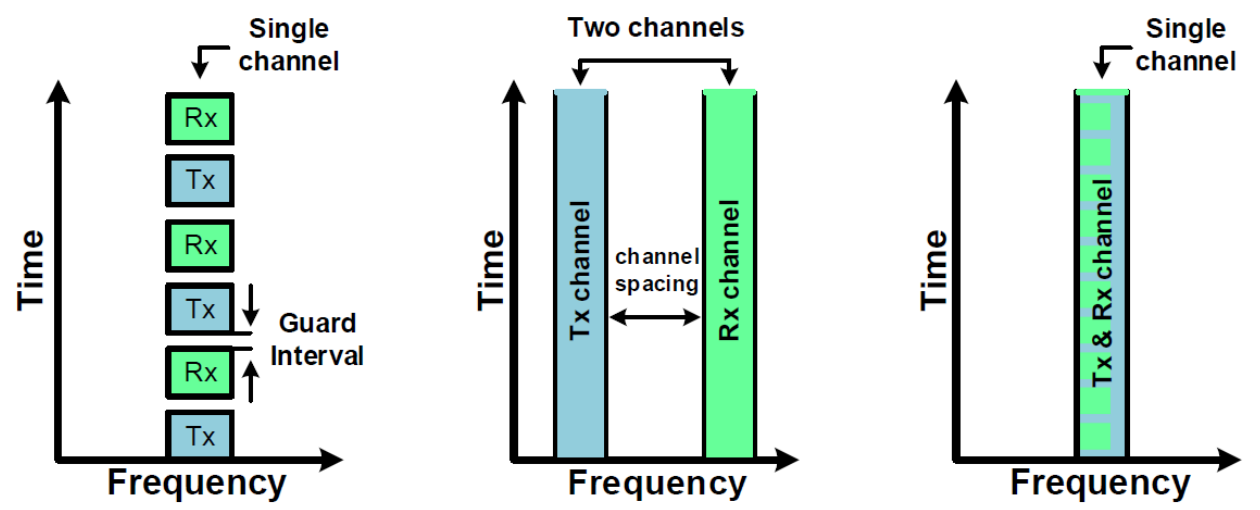

Figure 2.1: Spectrum and Time Utilization of FD Radios Compared to TDD and FDD [1]

\subsection{Full-Duplex Applications}

The obvious advantage for implementing FD radios over the conventional HD would be to double the spectral efficiency. Although there is a small amount of overhead that would prevent this from coming to fruition, it was shown in [4] that the spectral efficiency can be increased by $87 \%$ realistically. This also becomes more convincing for shorter range transmission networks like the migration from macro-cells to higher density small-cell base stations, because the TX powers are smaller and therefore more manageable. The main application for a standard FD radio without significant change to the protocol would be in WiFi networks where the nodes are stable and create the backbone for the network.

Besides the immediate benefit of an increase to network capacity, FD radios provide unique solutions to other problems that occur in present day network architectures. Namely, the hidden node problem can be addressed and almost be completely resolved. Here, if two transmitters are trying to send information to the same receiver simultaneously, there is no way of determining if the channel is free. The information being sent by both will collide at the receiver and will prevent the receiver from receiving either message. Instead, with FD radios, while a node is receiving information, it can also broadcast a message to notify other transmitters that the channel is being occupied [2,7].

Another application for FD radios is within relay stations. HD radios require an entire message to be received before it can be re-transmitted, delaying the transmission process proportionally to the number of hops in the relay chain. If FD radios are used instead, each node can start to transmit the received signal immediately $[2,7,8]$.

Overall, FD radios provide greater flexibility within communication networks and can 
reduce the frequency requirements. Achievable rate gains and future applications are currently being researched by many groups worldwide [4,9-11].

\subsection{Self Interference Cancellation}

In the past, the idea of FD wireless communication has been rejected because of the overwhelming power of the TX signal relative to the attenuated, desired RX signal. The strong TX signal can be several orders of magnitude larger than the incoming signal and will leak into the RX chain through the antenna interface creating an undesired SI signal. Not only will the RX signal be completely buried beneath the large SI signal without cancellation, but the SI signal can also have enough power to saturate the components in the receive (RX) chain. In particular, the analog-to-digital converter will saturate, deteriorating the SNR completely through the increase in quantization noise. This along with other nonidealities is discussed at the end of this chapter.

To achieve the advantages of FD over HD, as previously discussed, a large amount of SI cancellation is necessary. Ideally, this SI signal should be cancelled to below the original noise floor at the point of receiver decoding so that the SNR is not deteriorated from it. For example, if a TX signal in a WiFi network has an output power of $20 \mathrm{dBm}$ and the receiver noise floor is $-90 \mathrm{dBm}$, it follows that over $110 \mathrm{~dB}$ of SI cancellation is necessary for a FD radio to have good performance [5].

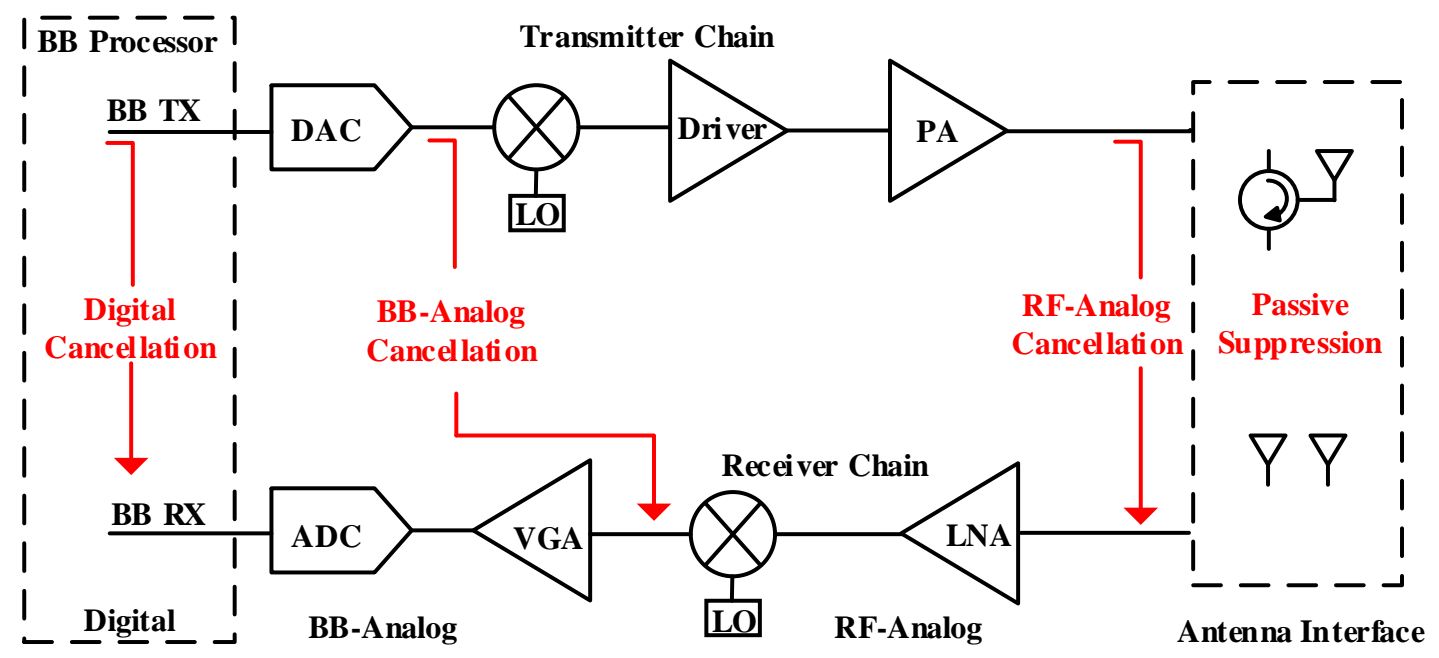

Figure 2.2: SI Cancellation Mechanisms Within a FD Radio [1] 
There has been a lot of research recently into different techniques to achieve this desired cancellation and they can be divided into three categories: passive suppression, digital cancellation, and analog cancellation depending on which section of the radio that the cancellation occurs. None of these techniques have been able to achieve full SI cancellation on their own using practical implementations. Therefore, a combination of all three techniques is used and are shown in Fig. 2.2.

There are many challenges surrounding each cancellation mechanism. The following subsections will introduce the cancellation mechanisms, the most prominent contributions relating to them, and their limitations.

\subsubsection{Passive Suppression}

Passive suppression in a FD radio refers to the isolation of the outgoing TX signal and the incoming RX signal at the antenna interface. Implementations of passive suppression incorporate multiple TX and RX antennas to suppress the SI signal at the input to the RX chain or a single, shared antenna with an improved duplexer used for isolation. Ideally, all of the cancellation would be done through passive suppression to avoid complexity in the receiver, but practical implementations only achieve around 20-30 dB of cancellation [4]. Tradeoffs between insertion loss, size, bandwidth, and isolation ability need to be analyzed to meet the required transmitter and receiver specifications.

Early into the investigation of FD radios, a common passive suppression techniques was to use separate antennas for the transmitter and receiver and create spatial isolation through the distance between them. The path loss of the TX signal through the air created the necessary cancellation $[9,12]$. This is a very simple method, but for most practical situations, it becomes obsolete as the area needed for cancellation of this type is very large. As an example, in [13], $70 \mathrm{~dB}$ of cancellation was achieved when the TX and RX antennas were separated by a distance of $5 \mathrm{~m}$. Base stations or WiFi nodes do not have this amount space available, let alone FD radios implemented in consumer electronics like laptops and smart-phones.

Another multi-antenna approach to passive suppression cancellation uses two TX antennas separated by a distance $d$ and $d+\lambda / 2$ away from the RX antenna as shown in Fig. 2.3. This creates a null for the center frequency at the RX antenna [2]. Although effective for narrowband TX signals, as the bandwidth increases the cancellation deteriorates at the band edges. Building upon the last approach, other researchers have implemented multiple TX antennas to create destructive interference at the RX antenna over a wider bandwidth [14]. This has the potential to be an effective method, but the far-field of the 
multiple transmitters needs be analyzed to ensure nulls are not created unintentionally and the receive signal is not suppressed as well [15]. Other antenna-based approaches include polarizing the TX and RX antennas to create minimal interference [16,17], creating antennas with higher directionality [1,12], and implementing beamforming techniques [12].

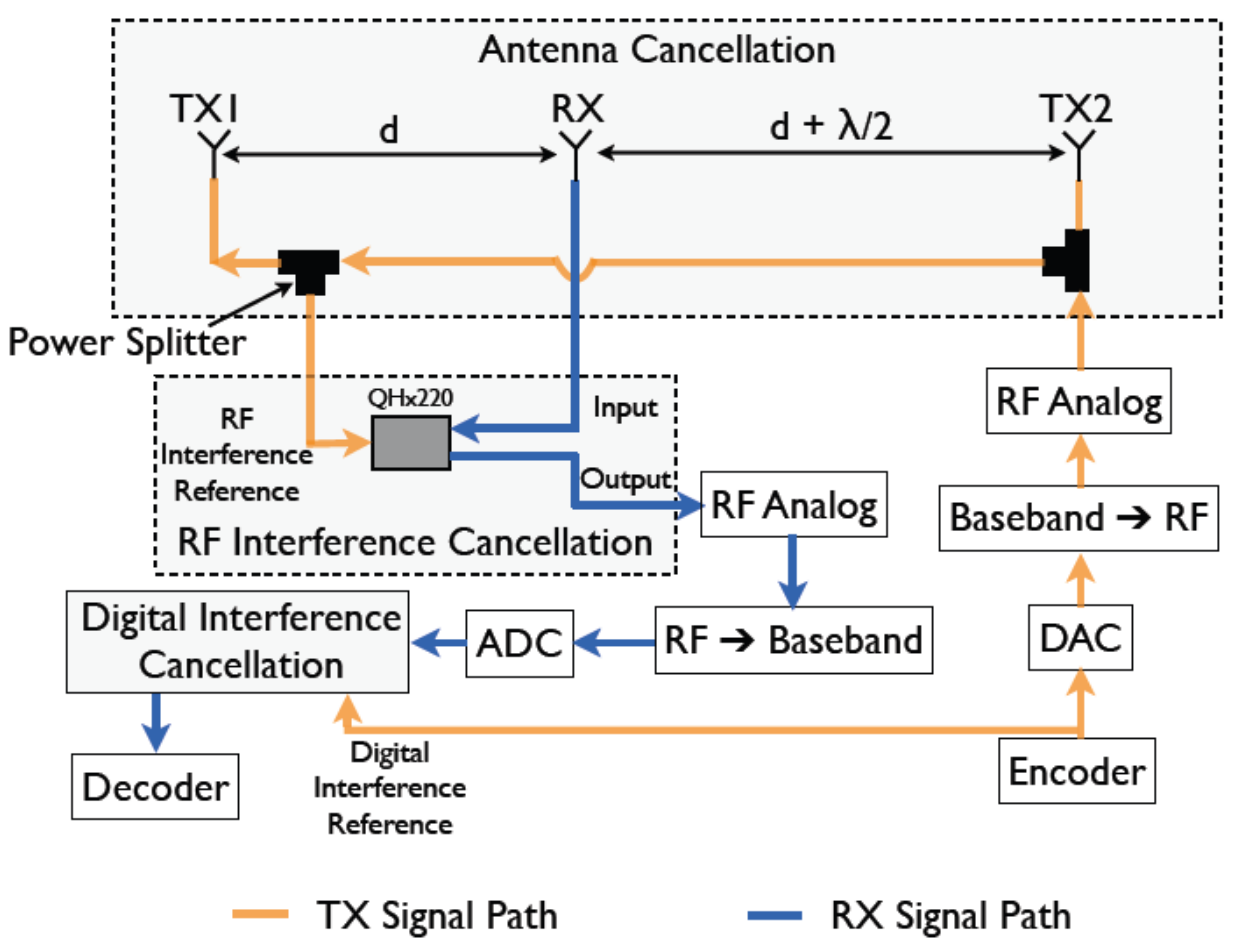

Figure 2.3: Passive Suppression Through Multiple Antennas [2]

The addition of a circulator before a single, shared antenna is a simple choice to isolate the the transmitter and the receiver $[4,7]$. A circulator is a ferrite device that provides directional routing to separate the outgoing and incoming signals. However, due to their wavelength dependent operation, they cannot be integrated for implementation in smaller electronic devices and can be quite expensive. They are also limited in their achievable bandwidth.

Instead, an alternate method for a single shared antenna application, referred to as an Electrical Balance Duplexer (EBD), has been realized in recent literature $[1,16,18,19]$. Here, the duplexer acts as a type of balun, which isolates the outgoing, balanced transmit signal from the receiver, but couples the incoming, unbalanced signal into the receiver. The standard EBD is shown in Fig. 2.4 with the transmitting operation on the left and the 
receiving operation on the right. This EBD implementation is able to achieve an isolation of $50 \mathrm{~dB}$ over a bandwidth of $6 \mathrm{MHz}$ [1]. The challenge of this type of duplexer lies in the adaptive matching network necessary to balance the transmit signal with the antenna input impedance, the inherent $3 \mathrm{~dB}$ insertion loss of the transmit signal, and the large insertion loss of the receive signal created from the coupling.

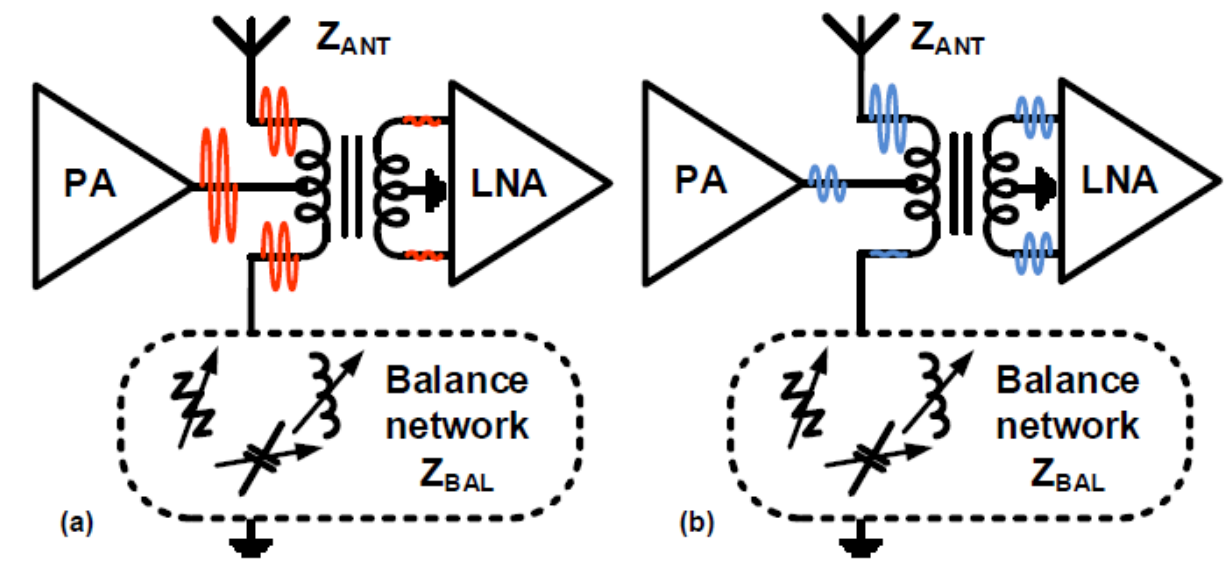

Figure 2.4: Transmit and Receive Operation of an Electrical Balance Duplexer [1]

Passive suppression techniques are the first line of defence in SI cancellation and can be chosen based on the particular application as they all have significant limitations. As well, due to their coordination with the antenna, they introduce a frequency dependency to the SI signal that will eventually need to be accounted for if wide modulation bandwidths are being transmitted.

\subsubsection{Digital Cancellation}

Digital cancellation is used to suppress the residual SI signal through digital signal processing in the receiver chain, after the analog-to-digital converter (ADC). By using the known TX digital signal, the SI signal can be replicated and subtracted from the received signal's digital samples, leaving only the signal of interest. Since digital cancellation occurs at the end of the RX chain, it is the last stage of cancellation before the RX signal is decoded.

SI channel estimation to produce this replica signal has been previously realized through the use of training symbols at the start of a TX packet along with the least squares algorithm to minimize the residual error [3]. The time domain response of the SI channel, 
which inlcudes the components from the digital-to-analog converter (DAC) through to the ADC, can be approximately emulated using a finite impulse response (FIR) filter in the digital domain assuming that the channel is linear [4].

Previous works have also used digital cancellation to compensate for nonidealities in the TX and RX chains from active components, and phase noise [4, 20-25]. Here, the linear and nonlinear distortions can be estimate using complex models to account for the dynamic TX signal through the components.

For successful implementation, digital cancellation is used after the SI signal has been sufficiently minimized so that the RX chain is not driven into saturation and the ADC can convert the incoming signal of interest without requiring an excessive number of bits. Quantization noise produced by a large SI signal compared to the desired RX signal cannot be cancelled through digital cancellation. This means that the SI signal should be cancelled so that it's power is on the order of the signal of interest at its minimum before the ADC. Digital cancellation also does not have the ability to cancel any thermal noise that leaks into the receiver from the transmitter. Typical cancellation achieved using digital cancellation is 30-50 dB [4], but it is also dependent on the amount of cancellation achieved beforehand. Using only passive suppression and digital cancellation will add strain and cost to the RX chain.

\subsubsection{Analog Cancellation}

Analog cancellation can be used to prevent the saturation of the receiver building blocks (low noise amplifier, mixer, ADC). It reduces the SI signal by sampling the transmit signal, inverting its phase, and adding it back into the receive chain before digitization.

If the sampled signal is combined with the receiver before the mixer, in the RF domain, it can be further categorized as RF-analog cancellation. Similarly, if it is combined after the receiver mixer, in the intermediate frequency (IF) domain, it is considered baseband-analog cancellation. However, it is important that enough cancellation is achieved before the low noise amplifier (LNA) to prevent saturation of this component. For this reason, RF-analog cancellation is critical and baseband-analog cancellation is only used as a supplementary technique.

Many RF-analog canceller topologies have been proposed over the last couple of years. In general, they can be further divided into two main methods: an auxiliary transmitter chain topology $[9,10,26]$ seen in Fig. 2.5 where the TX is sampled in the digital domain and a magnitude and phase adjustment topology [3], as shown in Fig. 2.6 where the TX signal is sampled in the RF-analog domain. 


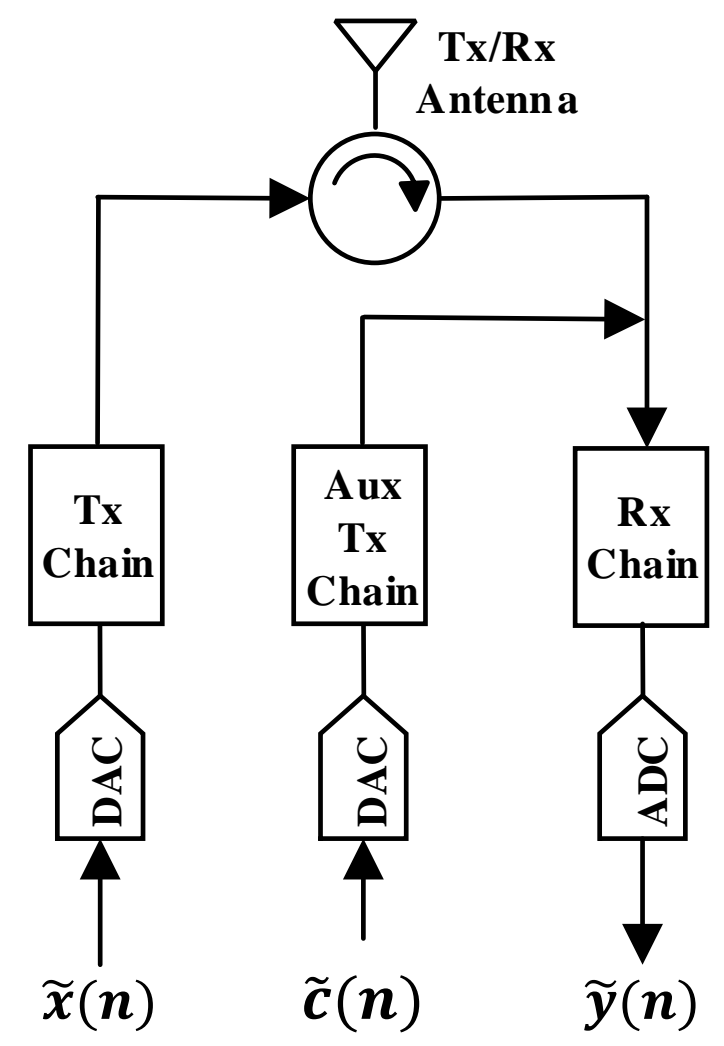

Figure 2.5: RF-Analog Cancellation Through Auxiliary TX Chain

In the former, the auxiliary TX chain generates a replica signal, which is out of phase with the SI signal $[9,10,26]$. This is demonstrated in [9], with $33 \mathrm{~dB}$ of cancellation over $625 \mathrm{KHz}$ from the auxiliary transmitter alone. However, to achieve good cancellation with this topology, the auxiliary transmitter requires accurate estimations of the various kinds of linear and nonlinear distortions in the main transmitter. Although it is feasible to identify these distortions, complex behavioural models are needed. In addition, both the auxiliary transmitter and the observation receiver are needed to operate at five times of the signal modulation bandwidth, which would significantly increase the overall power consumption of the radio.

In contrast, a magnitude and phase adjustment topology has gained popularity due to its simplicity in implementation. By taking a sample at the output of the PA, distortions of the TX are already embedded in the sampled signal. It also allows for the TX signal to be sampled at a higher power level, which results in less power consumption to produce the 


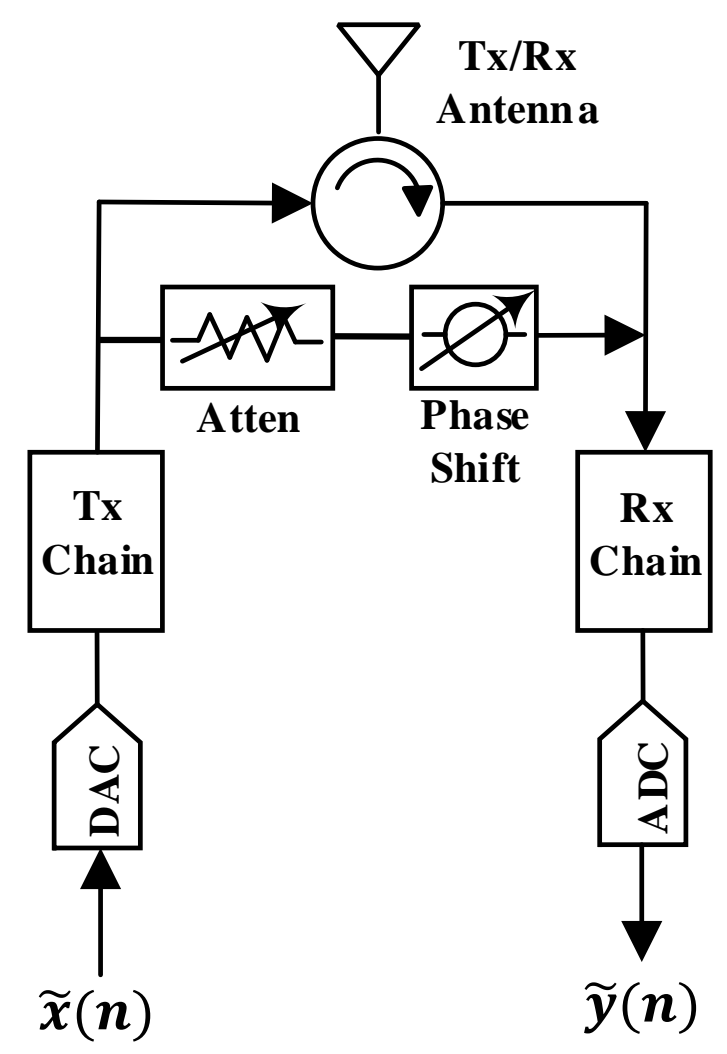

Figure 2.6: RF-Analog Cancellation Through Magnitude and Phase Adjustment

necessary cancellation signal. The magnitude and phase adjustment can be implmented through RF-analog components such as a phase shifter and attenuator [3] or a vector multiplier (VM) [27]. In [3], this magnitude and phase adjustment topology is used to achieve $25 \mathrm{~dB}$ of cancellation over $10 \mathrm{MHz}$. The block diagram of this implementation is seen in Fig. 2.7. However, a balun is used here instead of a simple coupler to produce the sample and phase inversion and minor adjustments are done through the attenuator and delay line.

While these topologies achieve a large amount of cancellation at the carrier frequency, the cancellation degrades rapidly as the modulation bandwidth broadens. This is mainly due to its inability to address the frequency dependent responses from the cancellation channel.

To address this frequency dependency, the concept of RF FIR filters is exploited [4,28]. Authors in [4] first augmented the topology of Fig. 2.6 with multiple magnitude and phase 


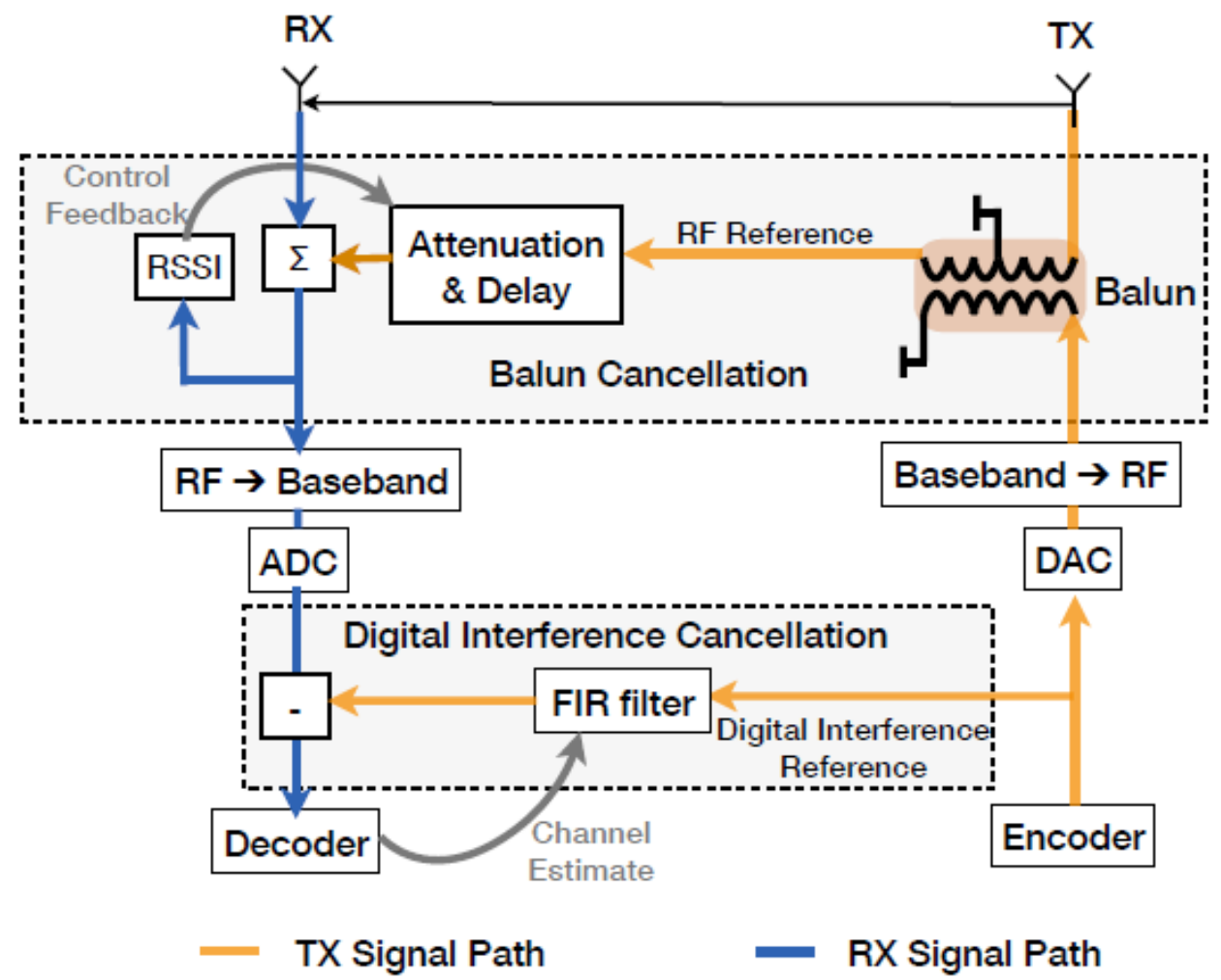

Figure 2.7: Implementation of General Magnitude and Phase Adjustment Cancellation [3]

adjust paths, referred to as taps. Each tap includes a fixed time delay line on the order of nanoseconds and a tunable attenuator, which is digitally controlled. Although this approach is effective in its cancellation, with $30 \mathrm{~dB}$ of cancellation over $80 \mathrm{MHz}$, the multiple taps, between 8 and 16 and the RF delay lines increase its size and complexity.

Alternatively, an RF-VM is used in [5] to replace the tunable attenuator [4]. The use of an RF-VM enabled finer resolution in controlling the magnitude and phase of each tap and an adaptive algorithm is developed to accurately adjust each of them. Hence, only 3 taps are needed to achieve similar cancellation performance as in [4]. However, each RF-VM adds significant complexity to the design and must be well calibrated to remove its in-phase and quadrature-phase (I/Q) imbalance. Moreover, two RF delay lines are still needed, the adaptive algorithm takes time to converge on the correct control, and multiple DACs and ADCs are used to establish these control voltages.

Authors in [29-31] proposed an entirely new, miniaturized, wide-band canceller topol- 


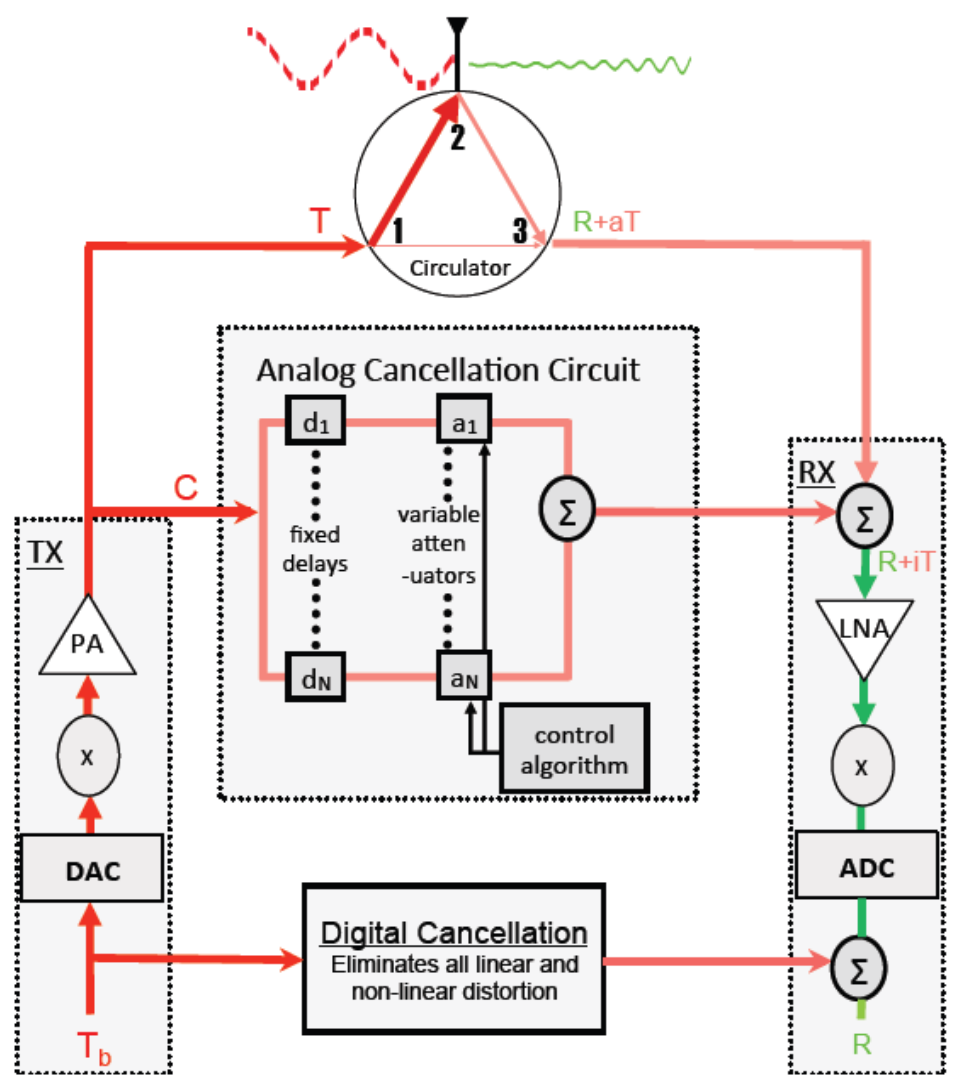

Figure 2.8: Implementation of Wideband RF-Analog Cancellation Through Multiple Taps of Variable Attenuators and Delay Lines [4]

ogy based on a bank of filters. These filters divide the modulation bandwidth into subbands, hence emulating a wide-band TX signal with multiple narrow-band signals. This approach eliminates the needs for RF delay lines, making it suitable for fully integrated circuits. However, the amount of cancellation demonstrated, $20 \mathrm{~dB}$ over a $20 \mathrm{MHz}$ bandwidth, is lower than the RF-FIR approach described previously.

All of the proposed RF-analog cancellers for wideband cancellation add undesired complexity to the overall system and there has yet to be a practical canceller developed with good cancellation performance. A new technique that improves upon these previous topologies is presented in Chapter 3. 

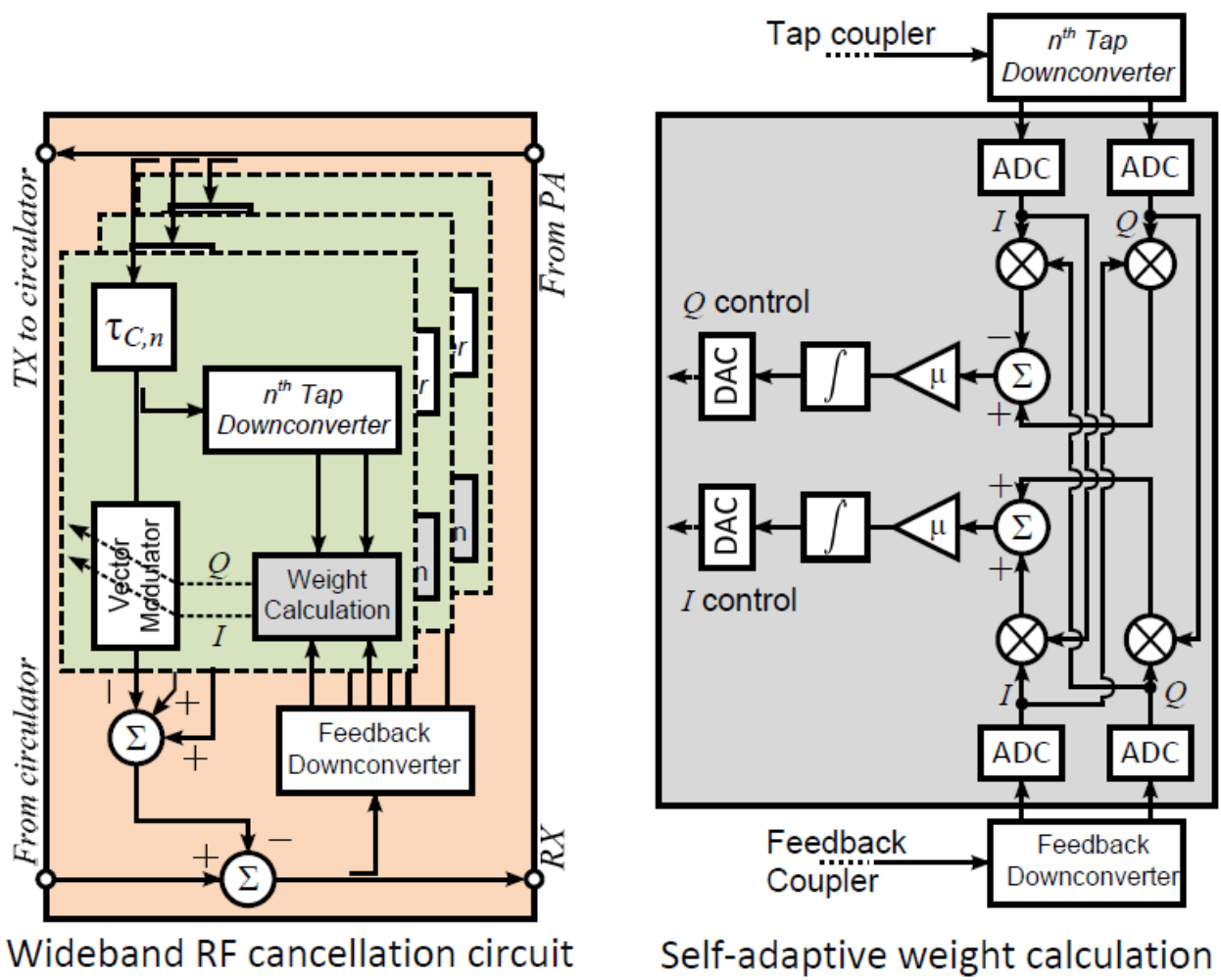

Figure 2.9: Implementation of Wideband RF-Analog Cancellation Through Multiple Taps of Delay Lines and Vector Multipliers [5]

\subsection{System Nonidealities}

Each cancellation technique has its own way of suppressing the SI signal, but the act of cancelling and the location of cancelling adds more noise to the system. There are common sources of noise that need to be optimized in the FD radio design and are discussed in this section. The following nonidealities of the system refer to Fig. 2.10 as a general transceiver with two common RF-analog cancellation options and a circulator for passive suppression to simplify the discussion.

\subsubsection{ADC Quantization Noise}

The ADC is designed to take the desired RX signal at the end of the RX chain and digitize it over its signal's entire dynamic range. When the SI signal is much larger than the desired 


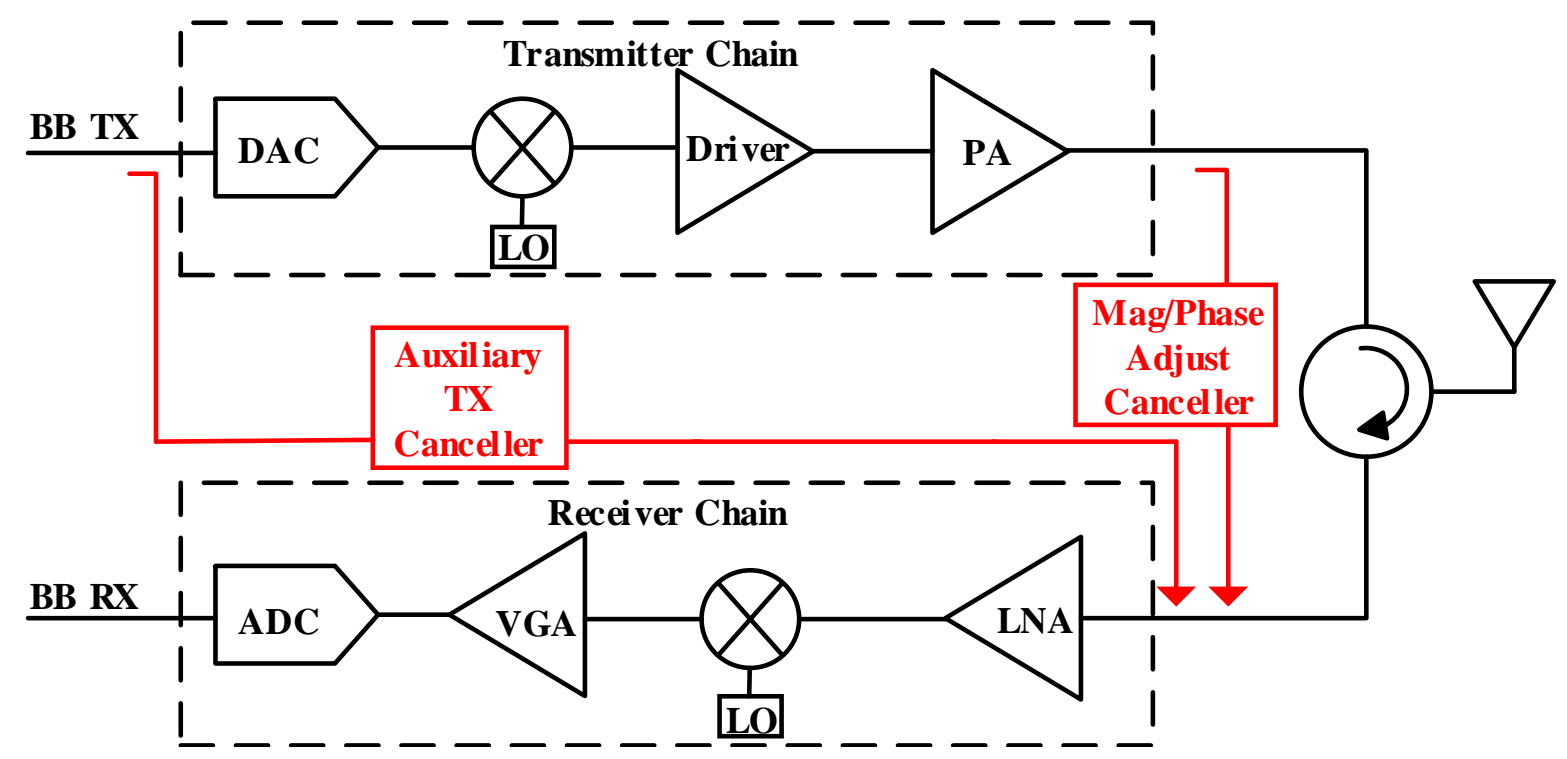

Figure 2.10: General FD Transceiver Topology For Impairment Analysis

signal, the majority of the ADCs dynamic range is used to quantize the SI signal and only a small portion is used to quantize the desired receive signal. With less bits effectively used to digitize the desired signal, the resolution of the ADC degrades and the quantization noise increases. The quantization noise adds to the total noise floor and reduces the SNR, leading to a degraded bit-error-rate (BER) [20].

Since quantization noise cannot be removed through digital cancellation, it is common for it to overwhelm the desired signal beyond recovery while implementing a FD radio. To ensure that this does not happen, more bits can be added to the ADC, to accommodate a larger SI signal. However, this is expensive and the practical limit for the number of bits is quickly reached. Instead, it is required that the SI signal is cancelled significantly before it reaches the ADC.

The amount of cancellation needed for a particular FD radio can be decided based on the number of bits available and the number of bits that can be spared for processing the strong SI signal. The amount of dynamic range available for a signal in terms of average power with $b$ bits is shown in (2.1). Here, it is assumed that the signal is split between two ADCs in a standard I/Q modulator and the peak-to-average power ratio (PAPR) of the signal in not included in the dynamic range [20,32].

$$
D R_{A D C, a v g}=6.02 b+4.76-P A P R(d B)
$$


The number of bits that would then be lost through quantizing the combined signal of interest (SOI) and the SI signal is formulated in (2.2) [20]. Here, $P_{S O I, \text { in }}$ represents the power of the signal of interest at the input of the receiver, $P_{S I, \text { in }}$ represents the power of the SI signal at the input of the receiver, and $P_{N, \text { in }}$ represents the thermal noise power at the input of the receiver. Lowercase letters represent the linear versions of the power and uppercase letters represent the powers in $\mathrm{dBm}$.

$$
\begin{aligned}
b_{\text {lost }} & =\frac{P_{t o t, A D C i n}-P_{S O I, A D C i n}}{6.02} \\
& =\log _{4}\left(\frac{p_{S O I, i n}+p_{S I, i n}+p_{N, i n}}{p_{S O I, i n}+p_{N, i n}}\right)
\end{aligned}
$$

This equation shows that the SI signal at the receiver input needs to be low compared to the desired signal for the dynamic range of the ADC to remain unchanged. As an example, if the SI signal is $20 \mathrm{~dB}$ larger than the SOI at the input to the ADC, the number of bits lost in the ADC is over three, which is a significant loss in bits. However, if it is $20 \mathrm{~dB}$ lower than the SOI, no additional bits are needed for the ADC to process the received signal. Other ways to reduce the number of bits lost would be to reduce the TX signal or increase the desired RX signal, possibly by reducing the range of transmission.

\subsubsection{Linearity of the Transmitter, Canceller, and Receiver}

Before the SI signal even reaches the ADC, it passes through other sensitive components in the RX chain like the LNA, mixer, and variable gain amplifier (VGA). These components operate in their linear region, specified individually by their $P_{1 d B}$ and $I P_{3}$ values. If enough power is present at their inputs, they will enter into the nonlinear region of operation, distorting the SI signal and the signal of interest in the form of harmonics or intermodulation distortions [33]. These distortions are difficult to cancel in later stages. As well, with very large input signals, the components can potentially be damaged. This is not usually a concern for the signal of interest alone as in a HD radio, but the high power of the SI signal can easily saturate the receiver.

Again, there are two solutions to ensure the components in the receive chain operate as they are intended to. First, the components of the receiver can be selected to have higher $P_{1 d B}$ and $I P_{3}$ values, extending the linear region to allow for a large SI signal power. This, however, has a practical limit. The other option is to cancel the SI signal through analog 
cancellation and passive suppression before reaching the sensitive receiver components. The latter is not simple to implement either as the increase in cancellation before the receiver will add complexity to the overall system and can be expensive.

The minimum required LNA $I I P_{3}$ value to keep the third order intermodulation products below the noise floor is defined as [33,34]:

$$
I I P_{3, L N A}=\frac{3\left(\operatorname{10log}\left(p_{\text {in, } \max }\right)\right)-F}{2}
$$

Where $F$ is the noise floor at the input of the LNA defined as:

$$
F=-174 d B m+10 \log (B W)+N F_{R X}
$$

And $p_{i n, \max }$ is the maximum input power to the LNA, defined as:

$$
p_{i n, \max }=p_{S I}+p_{S O I}
$$

The power of the SI signal will decrease with the amount of passive suppression and analog cancellation, therefore reducing the maximum input power to the LNA and relaxing its $I I P_{3}$ requirements.

Another source of nonlinearity in the system stems from the transmitter itself [35]. The transmitter is operating at a high power and it is not uncommon for the components to have small nonlinearities associated with them or in the case of the PA, fully operate in the nonlinear region to improve efficiency. Depending on the type of analog cancellation that is used, the auxiliary transmitter or the magnitude and phase adjustment, the TX nonlinearities may be accounted for in the cancellation. As well, the canceller may create undesired nonlinear distortions through its active components. The TX and RF-analog canceller nonlinearities are typically much smaller than the main SI signal and therefore aren't as concerning as the receiver saturation. The residual nonlinearities of the TX and RF-analog canceller can typically be estimated and cancelled in digital cancellation.

\subsubsection{Receiver Noise Floor}

When connecting the transmitter to the receiver, the SI signal power is not the only leakage power of concern. Noise from the transmitter will also be high enough to desensitize the received signal and estimation of the exact noise signal is not possible [21,36]. This becomes 
particularly relevant as the specification for transmitter noise is not very strict and can be much larger than the carefully specified noise floor of the receiver.

In the auxiliary TX topology, transmitter noise is a big issue and cannot be cancelled at later stages by digital or analog cancellation. However, previous literature has shown that by sampling the transmitter after up-conversion, like what is done in the magnitude and phase adjustment topology, the transmitter noise in the passive suppression path and the cancellation path are correlated and can be partially cancelled through analog cancellation. However, the cancellation path will also add extra noise to the receiver path.

The noise added from the transmitter and canceller circuitry in a general magnitude and phase adjustment topology can be analyzed using (2.6) from the analysis in [36].

$$
\begin{aligned}
p_{n}= & \left|g_{R X}\right|^{2}\left[F_{R X}+\left|a_{C R X}\right|^{2}\left(\left|g_{\text {canc }}\right|^{2} F_{\text {canc }}-1\right)-\left|a_{\text {ant }}\right|^{2}\right. \\
& \left.+\left|g_{\text {ant }}\right|^{2}\left|a_{R F}\right|^{2}\left|g_{P A}\right|^{2}\left(F_{P A}-1+F_{T X}\left|g_{T X}\right|^{2}\right)\right] p_{\text {th }}
\end{aligned}
$$

Where $g_{R X}$ and $F_{R X}$ refer to the voltage gain and noise figure of the entire receiver chain, which includes the LNA, mixer, VGA, and ADC. The PA gain and noise figure are represented by $g_{P A}$ and $F_{P A}$. The modulator, mixer, and driver gain and noise figure are represented by $g_{T X}$ and $F_{T X}$. The canceller, which includes magnitude and phase adjustment components, is represented by $g_{\text {canc }}$ and $F_{\text {canc }}$. The cancellation signal is combined in the receiver path through a coupler with an attenuation of $a_{C R X}$. The negative voltage gain through the antenna interface and the amount of RF-analog cancellation achieved on the noise are represented by $a_{a n t}$ and $a_{R F}$ respectively. Finally, the thermal noise power of the system is expressed as $p_{t h}$. These parameters are all in their linear forms.

The amount of RF-analog cancellation achieved and the amount of isolation through the antenna interface has a significant effect on the receiver noise floor. The individual components in the cancellation chain also add to the noise floor if they are not selected to have low noise figures.

\subsubsection{Oscillator Phase Noise}

Phase noise in the local oscillators of the system has been reported to be a major cause of concern in FD radios [24,37-39]. Local oscillators are used in the up-conversion and down-conversion of transmitters and receivers, respectively. In the case of the transmitter, 
the oscillator mixes with the baseband modulated signal, to create a modulated signal at the carrier frequency. If the local oscillator has phase noise, the carrier frequency has a small range of variation over time, distorting the expected TX signal.

For all cancellers, the amount of analog and digital cancellation that is achievable is inversely proportional to the variance of the phase noise [24]. However, the different analog canceller topologies lead to a different amount of achievable cancellation with this phase noise. The topologies of interest include analog cancellers that sample the TX signal before the mixer (referred to as pre-mixer like the auxiliary TX chain topology), and analog cancellers that sample the TX signal after the mixer (referred to as post-mixer like the magnitude and phase adjustment topology). The pre-mixer requires an additional oscillator to create the cancellation chain. This additional oscillator has uncorrelated phase noise with the oscillator in the TX chain, but can be matched to correlate the phase noise.

Results from [24] show that in the case of pre-mixer analog cancellers having unmatched local oscillators, the strength of the residual SI signal is dependent on the strength of the SI signal before cancellation. Therefore, at higher SI power levels, phase noise becomes a dominant source of residual SI. For pre-mixers that match the local oscillator in the TX path to the local oscillator in the cancellation path, allow for the correlation in phase noise helps to reduce the residual SI signal. Alternatively, post-mixer analog cancellers, which do not typically have a local oscillator in their cancellation path, can reduce the residual SI signal by simply reducing the error in channel estimation. The amount of cancellation does not depend on the SI signal strength. This provides a significant benefit for the magnitude and phase adjustment topology.

Finally, the transmitter and receiver local oscillators can also be matched to aid in the overall SI signal cancellation and improve the receive signal quality.

\subsubsection{Other Nonidealities}

Aside from the above nonidealities of the system there are other minor issues that need to be addressed and analyzed in a FD radio.

Primarily, I/Q imbalance and DC offsets in the I/Q modulator and demodulator can distort the signal and reduce the amount of cancellation if the cancellation relies on the known TX signal digital samples to replicate the SI signal. I/Q imbalance is an issue for both the receiver and the transmitter. However, I/Q imbalance and DC offsets do not vary significantly with time and can therefore be calibrated out of the system before operation [20]. The calibration for transmitter will be described later in Chapter 4 . 
The total power consumption of a FD radio is a concern for many practical implementations. Sampling at the output of the PA reduces the PA's power efficiency like in the case of the magnitude and phase adjustment topology. As well, the active components in the canceller like amplifiers and VMs will also add the overall power consumption of the system. However, the auxiliary TX chain topology, uses two additional DACs to replicate the SI signal, which are very power consuming especially if nonlinearities in the TX chain are added to the canceller estimation.

The antenna interface is also a difficult component to model in the system. Due to its inherent interaction with the surroundings of the radio, it becomes a dynamic component. A practical FD radio will be placed in a changing environment like weather in the case of a basestation or movement through a building in the case of a mobile phone. These environments create reflections of the transmitted signal that will add to the main SI signal and degrade the received signal of interest further [4]. Adaptive methods to account for these reflections are currently being researched. Since they are much smaller than the main SI signal, they will not be included in the scope of this thesis.

\subsection{Summary}

From the review in this chapter, it is evident that a lot of research has been done in the area of FD radio implementation. The critical piece of a FD radios to provide the necessary cancellation of the overwhelming SI signal without unnecessary stress to the system becomes the RF-analog canceller. It also is heavily relied on for cancellation of wide modulation bandwidth signals, accounting for frequency dependency in the antenna interface. The following chapters of this thesis incorporate these conclusions to build a new, optimal RF-analog canceller and measure its cancellation ability. 


\section{Chapter 3}

\section{Proposed Self-Interference Cancellation Approach: Theory and Design}

Based on the discussion in the previous chapter, it is critical to have enough RF-analog cancellation to suppress the SI signal before reaching the receive chain. It is also desirable to minimize hardware complexity so that components do not introduce more nonidealities into the system. This has yet to be seen in research for wideband RF-analog cancellers with good performance.

A novel digitally-assisted RF-analog self interference canceller is proposed in this chapter. The advantages gained by sampling the TX chain after the PA in the general magnitude and phase adjustment topology are exploited in the proposed technique. In addition, the new approach addresses the issue of cancellation over a wide modulation bandwidth while providing a simple and flexible hardware design. The following sections outline the system architecture, the algorithm for identifying the parameters of the system, and the implementation of this approach using off-the-shelf components.

\subsection{Proposed Digitally-Assisted RF-Analog SI Can- cellation Approach}

The proposed SI canceller follows many aforementioned RF-analog cancellers by employing the basic magnitude and phase adjust topology of Fig. 2.6. While previous works realized 
wide-band cancellation through the use of multiple RF taps, this increases the size of the canceller and leads to a significant increase in circuit nonidealities. Instead, the proposed cancellation approach overcomes this bottleneck by augmenting the basic, single tap topology with a low complexity, yet adequate, digital filter. This ensures flexibility and minimal hardware complexity, while maximizing the cancellation performance. A block diagram of the proposed FD radio, including the SI canceller, is illustrated in Fig. 3.1.

A single RF-VM dynamically adjusts the magnitude and phase of the sampled TX signal through a baseband control signal with fine resolution. An ideal block diagram of an RF-VM is shown in Fig. 3.2 and (3.1) expresses the ideal RF-VM operation with $y(n)$ representing the RF output, $x(n)$ representing the RF input, and $\tilde{v}_{I}(n)$ and $\tilde{v}_{Q}(n)$ representing the complex baseband control signal input. This input control signal is synthesized by processing the baseband components of the TX signal with the aforementioned filter.

$$
y(n)=x(n)\left(\tilde{v}_{I}(n)+j \tilde{v}_{Q}(n)\right)
$$

To derive the governing equation of the filter, a simplified behavioural model of the SI canceller is proposed. Fig. 3.3 shows its block diagram, which includes two FIR filters, $\mathbf{h}$ and $\mathbf{g}$, and the RF-VM. These FIR filters are used to model frequency dependent magnitude and phase responses of a linear path in the system using the difference equation in (3.2). An FIR filter in the RF-analog domain is implemented through multiple taps with attenuators and delay lines, similar to [4]. However, in the digital domain, an FIR filter can more easily be implemented through DSP techniques [40].

$$
y(n)=\sum_{l=0}^{M} b_{l} x(n-l)
$$

In this representation of the system, the FIR filter $\mathbf{h}$, models the overall frequency dependent magnitude and phase response of the isolation path, which includes the two couplers and the circulator. The other FIR filter $\mathbf{g}$ is used to model the frequency dependent magnitude and phase response of the cancellation path, which includes the two couplers and the post-amplifier placed after the RF-VM. The RF-VM acts as a linear element, performing a linear multiplication. The RF-VM differs from a nonlinear mixer element which introduces harmonics and intermodulation components into the spectrum. This arrangement of filters leads to a simple expression of the control voltage, $\tilde{v}(n)$, shown in the derivation below.

According to Fig. 3.3, the output signal, $y(n)$, is expressed as: 


$$
\tilde{y}(n)=\sum_{i=0}^{K_{1}-1} h_{i} \tilde{x}(n-i)+\left[\sum_{i=0}^{K_{2}-1} g_{i} \tilde{x}(n-i)\right] \tilde{v}(n)
$$

Where $K_{1}$ and $K_{2}$ denote the length of FIR filter $\mathbf{h}$ and $\mathbf{g}$, respectively, and $h_{i}$ and $g_{i}$ denote the $i$-th coefficient of FIR filters, $\mathbf{h}$ and $\mathbf{g}$, respectively. In the case of perfect cancellation, $y(n)=0$, the control signal can be derived from (3.3) as follows:

$$
\tilde{v}(n)=\frac{-\sum_{i=0}^{K_{1}-1} h_{i} \tilde{x}(n-i)}{\sum_{i=0}^{K_{2}-1} g_{i} \tilde{x}(n-i)}
$$

According to (3.4), the control signal that achieves perfect cancellation can be synthesized as a rational function FIR filter. The coefficients of the filter, $h_{i}$ and $g_{i}$, are initially unknown, but they can be identified using the identification process in the next section. 


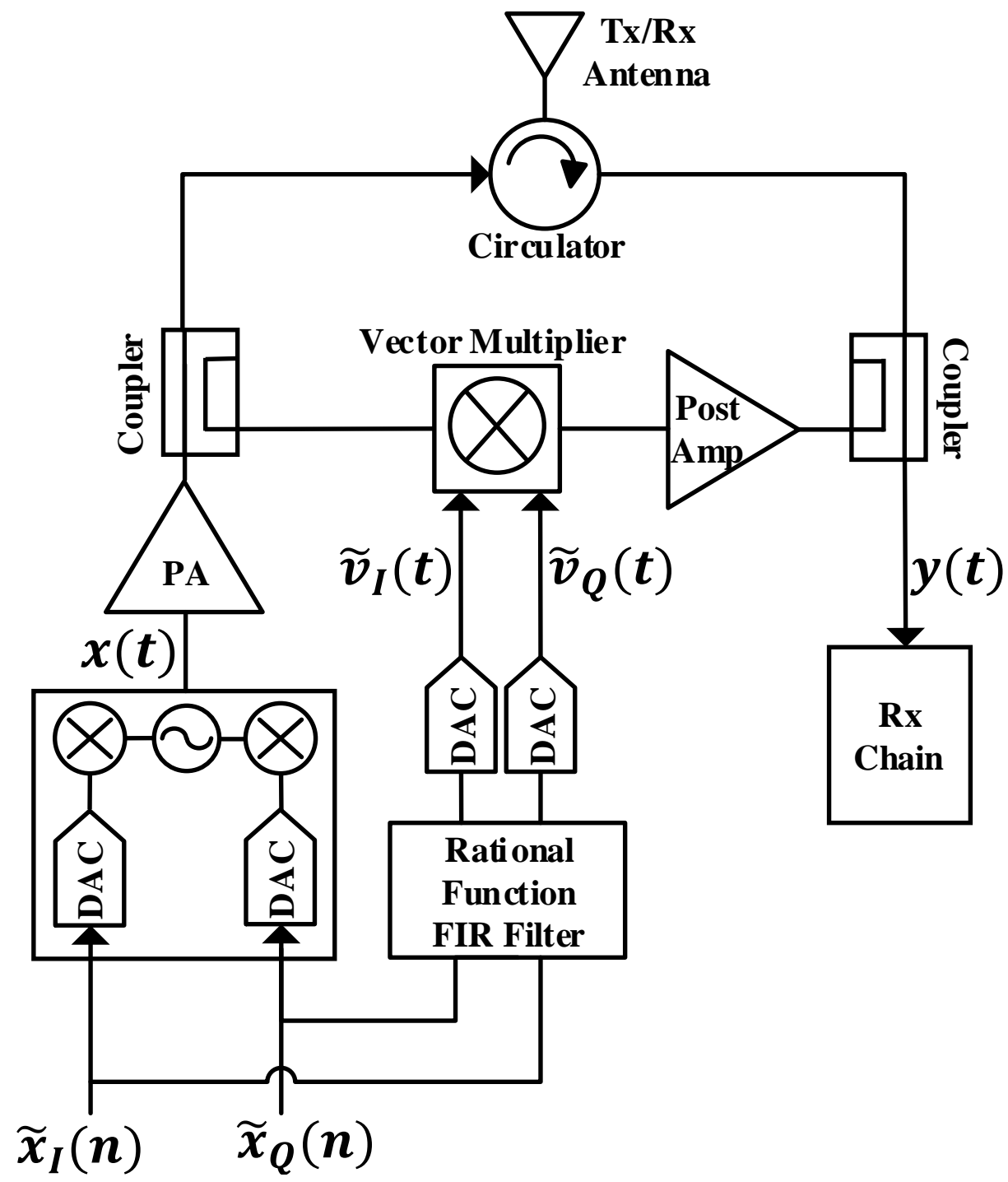

Figure 3.1: Block Diagram of the Proposed Digitally-Assisted RF-Analog Self Interference Cancellation 


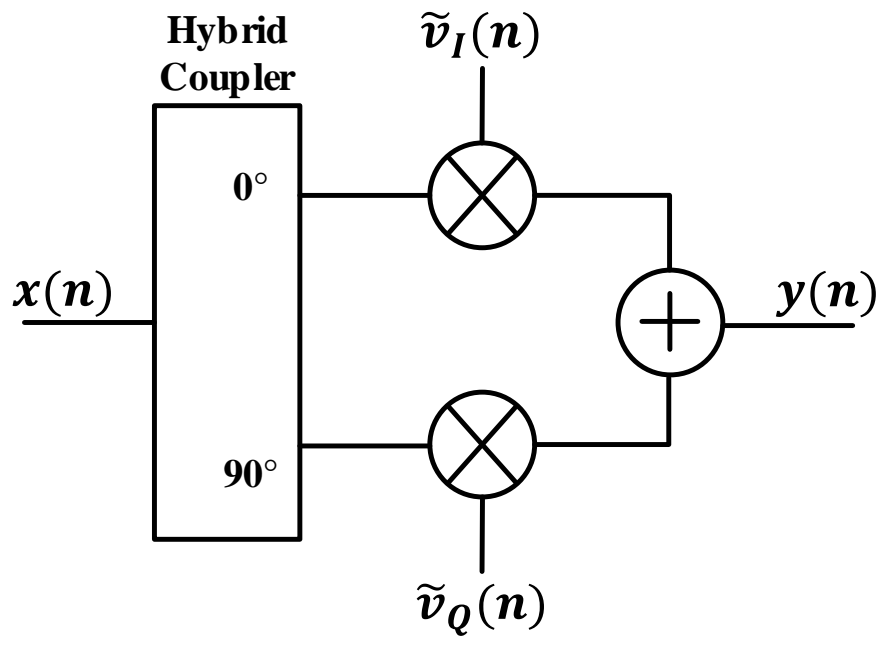

Figure 3.2: Block Diagram of an Ideal Vector Multiplier

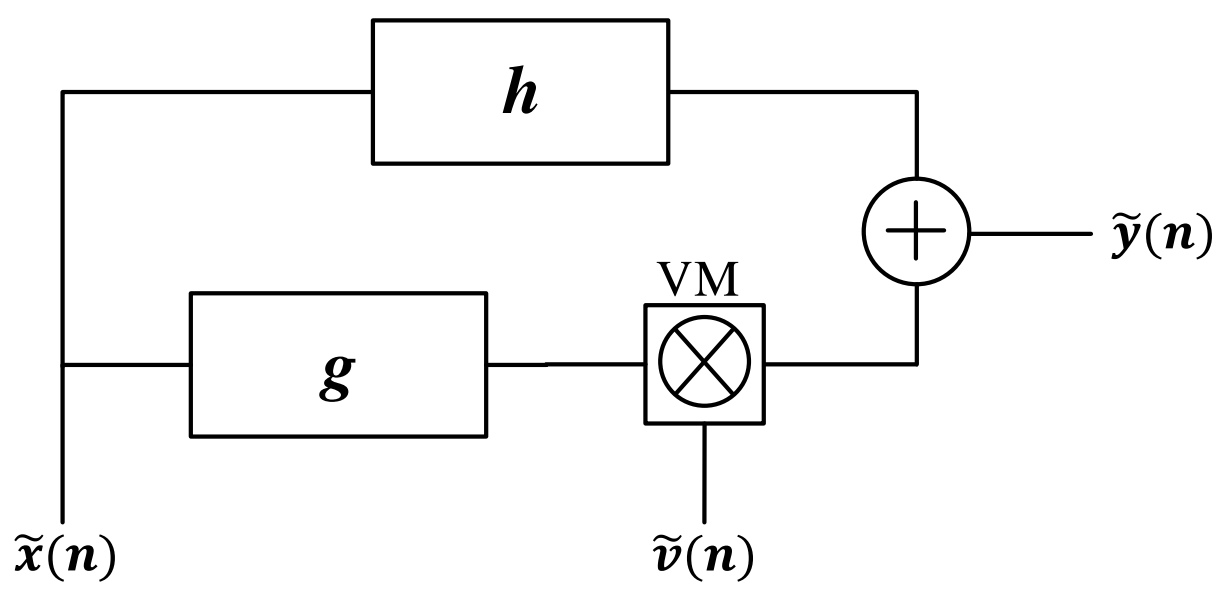

Figure 3.3: Baseband Equivalent Behavioural Model of the Full-Duplex Radio Front-End 


\subsection{The Proposed Parameter Identification Algorithm}

In this section, a linear algorithm to identify the coefficients of the proposed rational filter, i.e., $h_{i}$ and $g_{i}$, is proposed. The proposed deterministic algorithm requires only a single iteration, hence minimizing the effects of the parameter identification process on the transceiver. Referring to Fig. 3.1, the proposed canceller block diagram is a dual-inputsingle-output system. Using a total number of $\mathrm{N}$ samples of $\tilde{x}(n), \tilde{v}(n)$ and $\tilde{y}(n)$, a matrix form of (3.3) is derived:

$$
\mathbf{Y}=\mathbf{X} \times \mathbf{W}
$$

Where $\mathbf{X}, \mathbf{Y}$ and $\mathbf{W}$ denote the input signals matrix, the output signal vector and the unknown coefficients vector respectively. These can be individually described as follows:

$$
\begin{aligned}
& \mathbf{Y}=\left[\begin{array}{llll}
\tilde{y}(L) & \tilde{y}(L+1) & \ldots & \tilde{y}(N)
\end{array}\right]^{T} \\
& \mathbf{X}=\left[\begin{array}{cccccc}
\tilde{x}(L) & \ldots & \tilde{x}\left(L-K_{1}\right) & \tilde{x}(L) \tilde{v}(L) & \ldots & \tilde{x}\left(L-K_{2}\right) \tilde{v}(L) \\
\tilde{x}(L+1) & \ldots & \tilde{x}\left(L+1-K_{1}\right) & \tilde{x}(L+1) \tilde{v}(L+1) & \ldots & \tilde{x}\left(L+1-K_{2}\right) \tilde{v}(L+1) \\
\vdots & \vdots & \vdots & \vdots & \vdots & \vdots \\
\tilde{x}(N) & \ldots & \tilde{x}\left(N+1-K_{1}\right) & \tilde{x}(N) \tilde{v}(N) & \ldots & \tilde{x}\left(N+1-K_{2}\right) \tilde{v}(N)
\end{array}\right] \\
& \mathbf{W}=\left[\begin{array}{llllll}
h_{0} & \ldots & h_{K 1} & g_{0} & \ldots & g_{K 2}
\end{array}\right]
\end{aligned}
$$

Eq. (3.5) reveals the linear relationship with regard to its coefficients $h_{i}$ and $g_{i}$. As a result, a least squares error (LSE) algorithm can be applied to identify their values.

$$
\mathbf{W}=\left(\mathbf{X}^{T} \mathbf{X}\right)^{-1} \mathbf{X}^{T} \mathbf{Y}
$$

Ideally this identification algorithm is run once as the components remain static. In reality, there will be slight changes to the system with the input of a dynamic TX signal and especially after a real antenna is added. With this in mind, a coherence time for which the coefficients of the rational function FIR filter are relevant should to be established, dictating when the coefficients need to be updated. Previous research in [3] suggests that a realistic coherence time is on the order of ten seconds, which means that the identification time is 
required to be significantly shorter. This coherence time will also depend on whether the implementation is in a fairly static environment like a base station or WiFi node or a highly dynamic environment like a mobile device. The mobile device will have a much smaller coherence time. The identification time of the proposed approach has the advantage of not requiring iteration to settle on the correct coefficients, therefore, it should be a shorter period. The LSE algorthim finds the best fit to minimize the residual error in a single iteration and is also fairly robust to noise in the samples [3]. A tracking algorithm can also be developed to observe the deterioration of the SI cancellation, thus triggering the identification process of the isolation and cancellation path again, but is left out of the initial prototype that this thesis describes.

Another advantage to this new topology is that fixed delay lines are not necessary. Previously, delay lines were needed for each tap in the wideband cancellation topologies to replicate an FIR filter in RF-analog hardware. These delay lines rendered the topology impractical for real applications and prevented flexibility. Instead, the delay inherent to

the FIR filter, can be added in the digital domain because it is already included in the rational function FIR filter.

\subsection{Canceller Implementation}

A prototype of this proposed canceller approach is implemented using off-the-shelf components. It is important to validate the theory with non-ideal components to get a sense of whether it would be realistic, where the shortcomings of the design occur, and how much cancellation can be achieved beyond the theory and simulation. Connecting these components in such a manner will also add realistic component mismatch to the system. Fig. 3.4 shows the components used and their arrangement to mimic the block diagram of the system architecture in Fig. 3.1. This section will describe the components being used and a brief justification for their placement and values.

Instead of creating the RF signal from a high-speed arbitrary waveform generator (AWG), the I/Q modulator, ADL5375 from Analog Devices, is used to up-convert the baseband transmit signal to the chosen carrier frequency of $1.95 \mathrm{GHz}$. Using the upconverter will add the nonidealities discussed previously such as I/Q imbalance and DC offsets into the system and can be analyzed later as an impact to the system and cancellation ability. After being up-converted, the TX signal is amplified to a peak power of $23 \mathrm{dBm}$ at the output of the PA. The driver, ADL5320 from Analog Devices, provides $13.2 \mathrm{~dB}$ of gain and the PA, HMC455 from Analog Devices, provides $13 \mathrm{~dB}$ of gain. The $\mathrm{PA}$ and driver have not been pushed into the nonlinear region because their P1dB values 


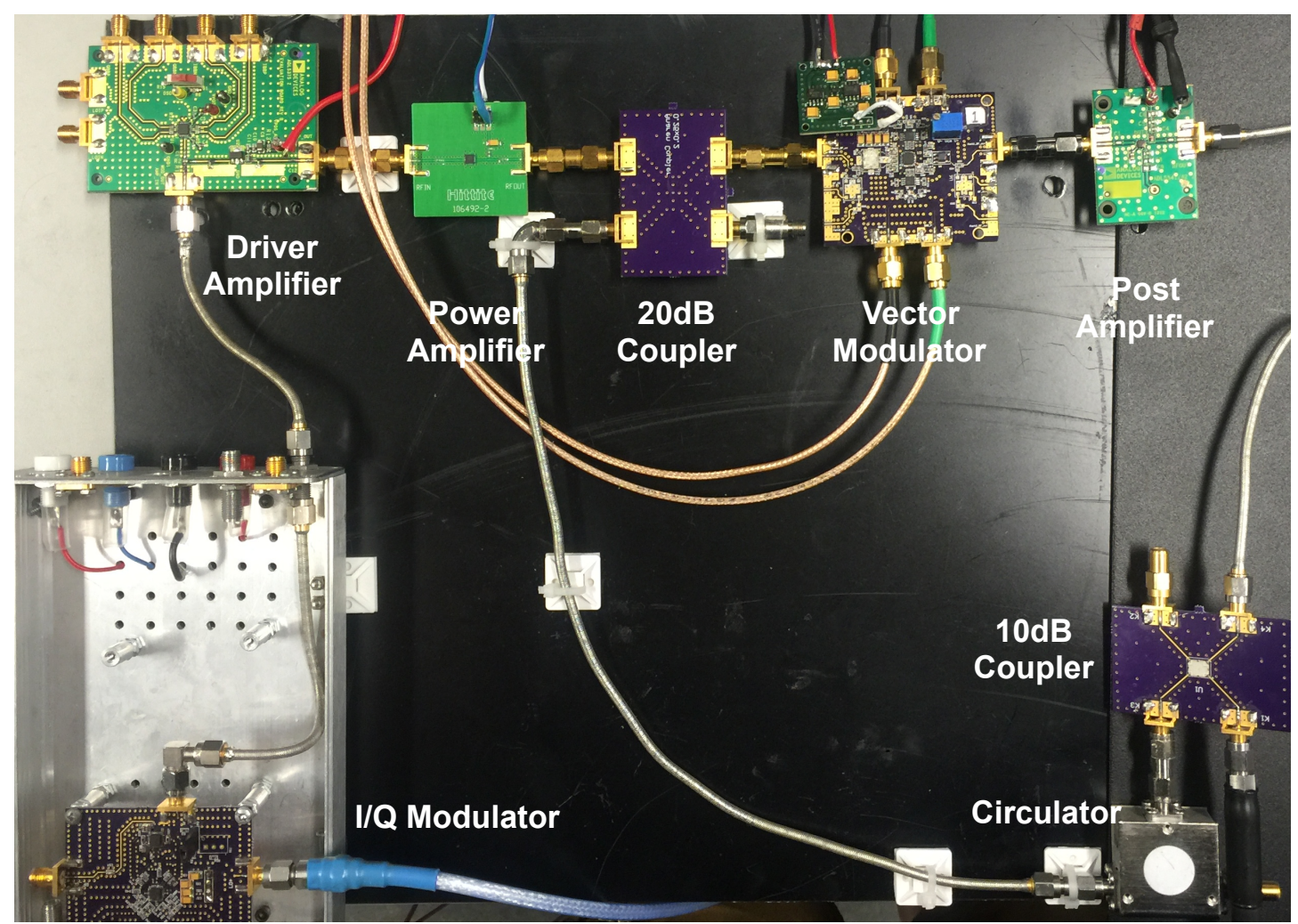

Figure 3.4: Experimental Setup of FD Radio Front-End Using Off-The-Shelf Components

are much higher that their peak output powers. If a nonlinear device is used, predistortion techniques can be implemented to meet the transmitter linearity specifications.

A simple circulator, MECA CN-1.950-GR, was used as the passive suppression technique, providing a fixed $23 \mathrm{~dB}$ of isolation while maintaining a frequency variation that would limit previous designs from cancelling the SI signal over wide modulation bandwidths. The circulator is terminated with a $50 \mathrm{ohm}$ impedance at the output antenna port. This is another simplification made for this first prototype to neglect the effects of antenna mismatch and reflections from a dynamic environment.

The canceller is simple in design and is made up of a coupler to sample the TX signal, a RF-VM to adjust the magnitude and phase, a post-amplifier to achieve the necessary power for cancellation, and another coupler to combine at the input of the receiver. The tradeoff discussed in Chapter 2 to achieve the optimal power, noise, and linearity performance while using the given input range for the control signal is analyzed. Values for the couplers, the post-amplifier, and the RF-VM gain settings are altered until the best performance with 
Table 3.1: Active Component Parameters for the Proposed Canceller Experimental Setup

\begin{tabular}{cccccc}
\hline Component & Part Number & Gain $(\mathrm{dB})$ & OP1dB $(\mathrm{dBm})$ & OIP3 $(\mathrm{dBm})$ & $\mathrm{NF}(\mathrm{dB})$ \\
\hline \hline Driver & ADL5320 & 13.2 & 25.7 & 42 & 4.4 \\
PA & HMC455 & 13 & 27.5 & 42 & 6 \\
Post-Amp & ADL5541 & 14.7 & 16.3 & 39.2 & 3.8 \\
RF-VM & ADL5390 & - & 10.2 & 20 & - \\
\hline
\end{tabular}

the available components is achieved. A $20 \mathrm{~dB}$ coupler, IP520S from Anaren, is used to sample the TX signal, providing lower insertion loss after the PA in the transmitter (and therefore higher PA efficiency) and allowing for higher power at the PAs output without saturating the VM. It is important that the VM remains in its linear region for it to be modelled as a linear multiplication. This unfortunately means that the input to the VM should not go above $3 \mathrm{dBm}$ of peak power. Future designs could improve upon this specification to optimize the FD radio. Analog Device's ADL5390 VM is chosen as a result of its good performance previously characterized in the lab. The post-amplifier not only creates the necessary gain for cancellation, it also dictates the control signal's average, complex value. This is important because there are voltage bounds to which the input of the VM can allow. Although, the control signal does not have a very large variation, it should still comfortably sit in the middle of these bounds. Anaren's IP510S $10 \mathrm{~dB}$ coupler is chosen as a tradeoff between insertion loss in the receiver and gain needed from the post amplifier. Table 3.1 and 3.2 summarize the important component specifications of the implemented transmitter and canceller.

Table 3.2: Passive Component Parameters for the Proposed Canceller Experimental Setup

\begin{tabular}{ccccc}
\hline Component & Part Number & $\begin{array}{c}\text { Insertion } \\
\text { Loss }(\mathrm{dB})\end{array}$ & $\begin{array}{c}\text { Coupling } \\
\text { Factor }(\mathrm{dB})\end{array}$ & Isolation (dB) \\
\hline \hline 20 dB Coupler & IP520S & 0.2 & 19.8 & - \\
10 dB Coupler & IP510S & 0.65 & 9.5 & - \\
Circulator & CN-1.950-GR & 0.5 & - & 23 \\
\hline
\end{tabular}

The receiver was not added to this initial prototype to help simplify the analysis of the transmitter, the isolation path and the cancellation path. As well, the receiver was removed to achieve accurate measurements of the SI cancellation. 


\section{Chapter 4}

\section{RF Canceller Measurement Results}

This chapter discusses the validation process of the initial RF-analog SI canceller prototype. The theory presented earlier will be tested using various TX signals to verify its cancellation ability over wide modulation bandwidths, high power, and with realistic components. The measurement equipment and the calibration process are also reviewed in the first sections for a complete description of the verification process. The setup is initially tested using a continuous-wave (CW) signal ensuring accuracy in the VM's magnitude and phase resolution. Then wider modulation bandwidth signals are used to determine the system's potential for cancellation and its limitations. Previous research has achieved a maximum cancellation of $40 \mathrm{~dB}$ over $80 \mathrm{MHz}$ from the RF-analog SI canceller. However, the previous topologies have more complexity in their analog components, adding major nonidealities to the system.

\subsection{Measurement Setup}

The cancellation ability of the proposed digitally-assisted RF-analog SI canceller, shown in Fig. 3.1, is assessed here using off-the-shelf components in a FD radio configuration. A complete experimental setup is devised as shown in Fig. 4.1.

The baseband source signal driving the TX chain is generated using an arbitrary waveform generator (AWG), Keysight N8241A. The AWG takes in-phase and quadrature-phase digital samples and converts them to a baseband-analog signal using 15-bit digital-toanalog converters and an I/Q bandwidth of $1 \mathrm{GHz}$. Another AWG, Keysight N8241A with the same specifications, is used to produce the baseband control signal for the VM after 


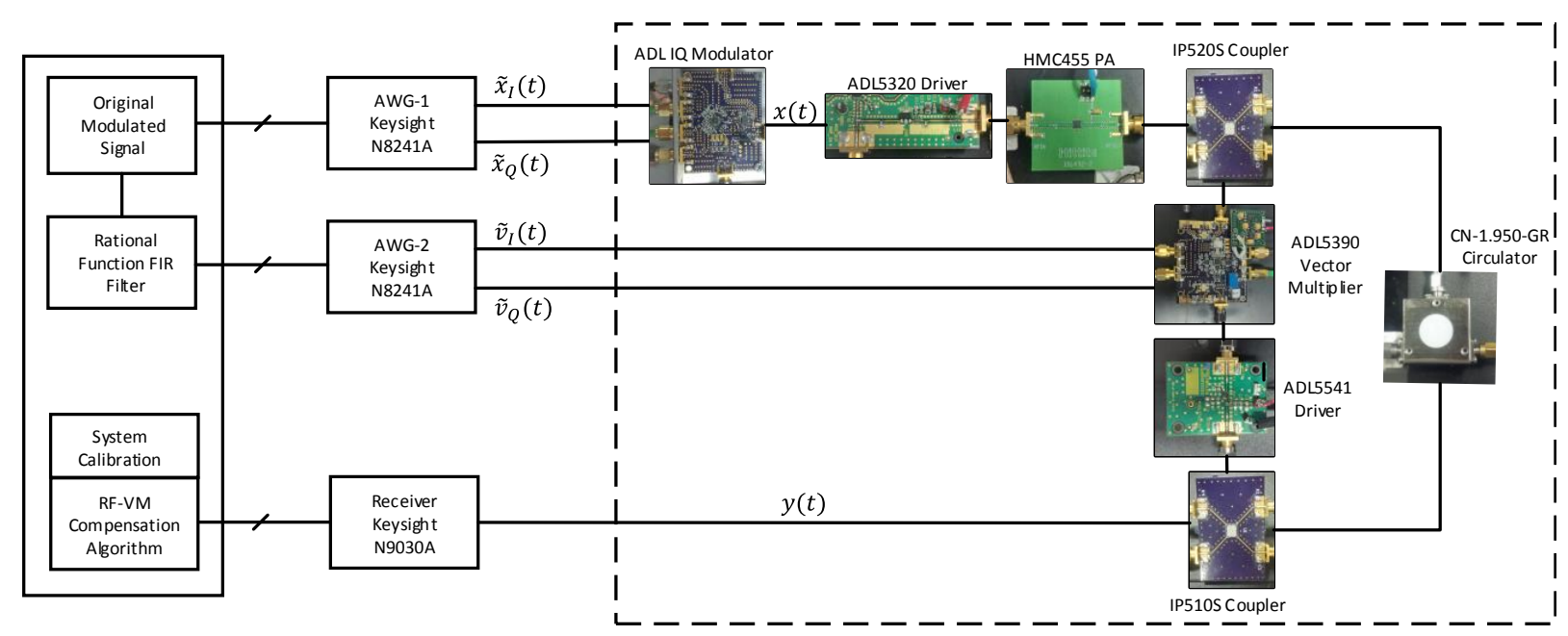

Figure 4.1: FD Radio Equipment Setup and Connection

going through the identification process outlined in Chapter 3. Here, the resolution of the signal is very important to create the correct magnitude and phase adjustment for the VM. Error in quantization can lead to error in cancellation and add noise to the system. It is important that the AWG has enough bits in its DACs to minimize the cancellation error and quantization noise. For every bit added to the AWG's DAC, the number of voltage levels doubles for the generated waveform [41]. The amount of cancellation error with this AWG setup is tested by cancelling a CW signal in Section 4.1.2. These AWGs are synchronized through Matlab software to ensure the control signal is properly time-aligned with the sampled TX signal at the input of the RF-VM.

The Keysight N9030A vector signal analyzer (VSA), replaces the RX chain to capture a signal with up to $160 \mathrm{MHz}$ of modulation bandwidth using two 16-bit ADCs. This allows for a measurement of the cancellation from the RF-analog SI canceller alone. There is a tradeoff between bandwidth and dynamic range for the ADCs in the VSA. To accomplish measurements of wide modulation bandwidth signals, the bandwidth of the anayzer itself must increase, which typically results in less bits to quantize the signal. With less bits, the quantization noise increases and the amount of cancellation can be buried beneath this noise. For the signals being tested in this case, the $160 \mathrm{MHz}$ VSA is sufficient. The VSA is used as a spectrum analyzer to capture the spectrum of the SI signal as well as collecting the full baseband I/Q data for further processing.

All of the equipment is connected through GPIB cables to the computer and are controlled using Matlab software. Matlab scripts establish the settings for each piece of 
equipment, upload the TX and control signals, trigger their transmission, and capture the output. The control signal filter parameters are also identified in Matlab once the initial samples have been collected.

\subsubsection{Calibration of Measurement Setup}

Although the off-the-shelf components are ready to use, they have imperfections associated with them. In particular, the I/Q modulator and the VM have inherent distortions from their I/Q branches, which can fortunately be calibrated out before use because they are approximately static over a long period of time $[42,43]$.

The TX I/Q modulator takes the baseband in-phase and quadrature-phase of the TX signal in separate branches at the output of two DACs and mixes them with a local oscillator. This modulates the baseband data onto the carrier frequency. These branches typically have unwanted DC offset errors, creating a carrier feedthrough component in the frequency domain. The branches can also have mismatches in their gain and phase. These imperfections will degrade the error vector magnitude (EVM) of the signal constellation, which eventually leads to a higher BER [42]. A calibration of the I/Q modulator is done in two steps, to isolate the offsets and imbalances. A single sideband spectrum is applied, where the I and Q signals are driven with cosine and sine signals respectively. This produces a desired sideband signal, a carrier-feed-through signal from the offsets, and an undesired sideband signal from the gain and phase imbalances. The offsets is corrected by minimizing the carrier-feed-through signal. Once the offsets are resolved, the imbalances are corrected by minimizing the undesired sideband. The frequency dispersive analog circuitry is modelled with four FIR filters for the imbalance terms [43]. These offsets and the imbalance matrix are saved and applied before use of the I/Q modulator.

The RF-VM can also be calibrated in a similar manner. However only a narrowband calibration is done to simplify the calibration process and ensure that the implementation remains practical. The DC offset voltages and the I/Q imbalances are still corrected, but four real constants are used for the imbalance terms instead of the FIR filters, therefore the frequency dependency of the components are not taken into account [43].

Finally, the third step in the system calibration is the time alignment between the sampled TX signal at the input of the RF-VM and the control signal for the RF-VM. This is determined only after the corrections for the I/Q modulator and the RF-VM have been implemented. Here, a cross-correlation between the two signals, varying the delay in

the TX signal, is done, establishing the amount of delay that needs to be added before the system can be used. This alignment allows the correct control signal at one point in 
time to be multiplied with the sampled TX signal for complete cancellation. The delay is recorded and added through Matlab software to the settings of the AWG for the TX signal. A significant misalignment between the signals will lead to errors in the SI cancellation.

\subsubsection{CW Signal Cancellation}

A CW signal is initially transmitted to analyze the cancellation ability of a signal with a narrow modulation bandwidth. It also gives an indication of the resolution of the VM in creating an accurate magnitude and phase adjustment and the resolution of the AWG in creating the correct control signal.

With the transmission of a CW signal, cancellation occurs only at one frequency. Therefore, there is no need to implement the full rational function FIR filter. Instead, the real and complex input signal to the VM can be implemented with constant voltages, to replicate the magnitude and phase adjustment at this center frequency. An iterative method is used to find the optimal magnitude and phase of the complex control signal minimizing the SI signal.

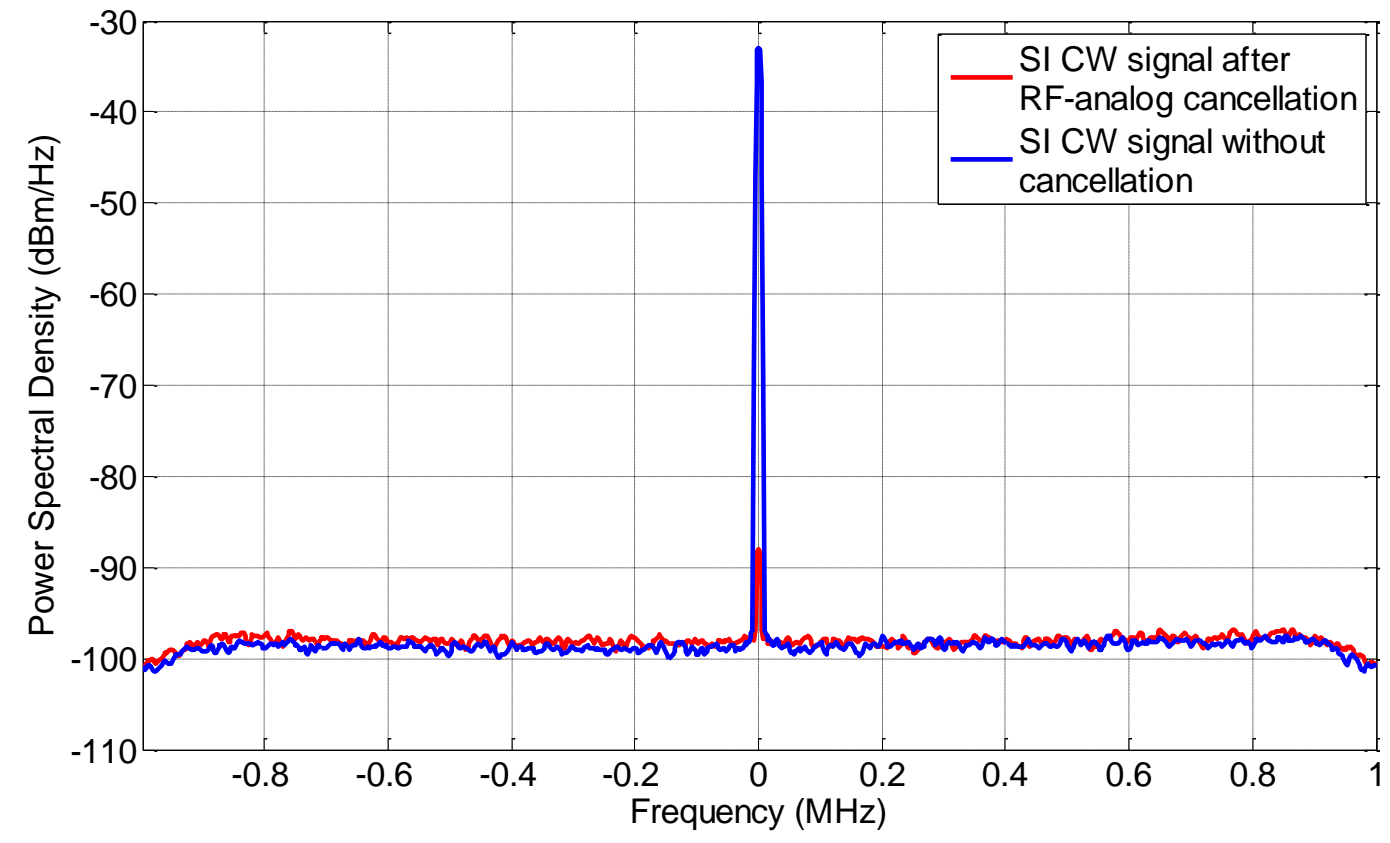

Figure 4.2: RF-Analog SI Cancellation of CW Signal with Constant Voltage Control Signal 
Ideally, the SI signal should be cancelled entirely to the noise floor of the receiver, but Fig. 4.2 shows that only $55 \mathrm{~dB}$ of cancellation is achieved for a $\mathrm{CW}$ signal. This puts an upper limit on the amount of cancellation that should be expected for wider modulation bandwidths. This less then ideal cancellation is due to the nonidealities of components and the cancellation resolution.

\subsubsection{TX Test Signals}

After proving that the VM has the desired resolution for cancelling the SI signal, the full prototype is tested using modulated TX signals. Although the theory does not limit the modulation bandwidth of the TX signal, the signal analyzer has a bandwidth constraint

of $160 \mathrm{MHz}$. The TX signal should have a modulation bandwidth even smaller than the VSA's constraint because multiplication by the VM will extend the cancellation signal's bandwidth.

Four different TX signals were used with bandwidths ranging from $20 \mathrm{MHz}$ to $120 \mathrm{MHz}$. The signals are as follows:

1. A single-band formed by a four-carrier WCDMA signal with a total bandwidth of $20 \mathrm{MHz}$ and a PAPR of $7.14 \mathrm{~dB}$.

2. A dual-band formed by a three-carrier WCDMA signal and a $15 \mathrm{MHz}$ LTE signal with a total bandwidth of $40 \mathrm{MHz}$ and a PAPR of $8.4 \mathrm{~dB}$.

3. A tri-band formed by a four-carrier WCDMA signal, a $15 \mathrm{MHz}$ LTE signal, and a $20 \mathrm{MHz}$ LTE signal with a total bandwidth of $80 \mathrm{MHz}$ and a PAPR of $10.9 \mathrm{~dB}$.

4. A tri-band formed by a two-carrier WCDMA signal, a $15 \mathrm{MHz}$ LTE signal, and $10 \mathrm{MHz}$ LTE signal with a total bandwidth of $120 \mathrm{MHz}$ and a PAPR of $9.1 \mathrm{~dB}$.

\subsubsection{Control Signal For Training}

A temporary control signal is used for the parameter identification process. This allows for the determination of the FIR filter coefficients to model the isolation path and cancellation path. Once these have been found, they are used to establish a new control signal that will completely cancel the SI signal when applied to the input of the VM. The initial control signal that is chosen will have a slight effect on the cancellation outcome. 
As mentioned earlier, the cancellation signal bandwidth increases after the multiplication of the sampled TX signal and the control signal. An increase in the control signal bandwidth, increases the output signal bandwidth. For this reason, the control signal is chosen to have a modulation bandwidth of one tenth of the TX signal.

The identified control signal will also be dependent on the number of taps used for the FIR filters. The number of taps is empirically chosen and it is determined that only three taps are needed to achieve good cancellation performance with the tested modulation bandwidths. This significantly reduces the complexity and shortens the identification time.

Finally, due to the constraints of the VM input, the control signal needs to be well within the specified input range of the $\mathrm{VM},+/-0.5 \mathrm{~V}$. This means that the average values of the complex control signal's voltages should be close to the middle of this range. The gain of the post-amplifier and the gain setting on the VM are selected to ensure that the correct range for the control signal is established. Due to the division of the rational function FIR filter, it is possible to get outlier control signal values beyond the VM input range and therefore clipping of the control signal must be done. This will add to cancellation error in the system if the control signal is clipped too much. When the system is properly setup, outliers do not occur frequently so their effect on the cancellation is minimal. For this implementation of the FD sytem, the control signal has a variation of approximately $20 \%$ of the VM's input voltage range.

\subsection{Measurement Results}

Fig. 4.3,4.4, 4.5, 4.6 depict the power spectral density of the signals obtained at the output of the SI canceller, denoted by $y(t)$, in Fig. 4.1. These figures highlight the level of SI signal for three different scenarios: with passive suppression only, with passive suppression and RF-analog cancellation using the general magnitude and phase adjustment technique (as shown in Fig. 2.6), and finally with passive suppression combined with the proposed digitally-assisted RF-analog cancellation approach. These measurements were conducted while maintaining a peak power of $23 \mathrm{dBm}$ at the PA output. Furthermore, the order of each FIR filter was set to three, minimizing the underlying hardware complexity.

To implement the general magnitude and phase adjustment cancellation technique, a control signal, similar to that of the CW cancellation, is used with the same hardware setup as Fig. 3.4. Here, the cancellation is optimized for one frequency only, which works well for narrowband cancellation, but degrades over wider modulation bandwidths. It becomes obvious from Fig. 4.3, 4.4, 4.5, 4.6 that as the frequency deviates from the center frequency, 
the cancellation degrades. This cancellation scheme becomes practically ineffective for TX signals with over $80 \mathrm{MHz}$ of modulation bandwidth.

According to Fig. 4.3, the proposed method with the implementation of the rational function FIR filter, maintained excellent SI cancellation of $50 \mathrm{~dB}$ over a $20 \mathrm{MHz}$ signal bandwidth. It represents an improvement of about $20 \mathrm{~dB}$ cancellation compared to the magnitude and phase adjustment RF-analog SI cancellation approach. This brings the combined SI cancellation obtained using passive suppression and the proposed digitallyassisted, RF-analog approach to $73 \mathrm{~dB}$. This significant reduction in the SI signal will allow digital cancellation to reach the required total SI cancellation for a successful FD radio.

Fig. 4.3, 4.4, 4.5, 4.6 demonstrate the ability of the proposed cancellation technique to maintain excellent SI cancellation of higher than $40 \mathrm{~dB}$ for test signals with modulation bandwidth of up to $120 \mathrm{MHz}$. It is a significant improvement from the general magnitude and phase adjustment approach. This also confirms the advantage of the proposed cancellation approach in addressing the frequency-dependent behaviour of the isolation path with minimal hardware complexity.

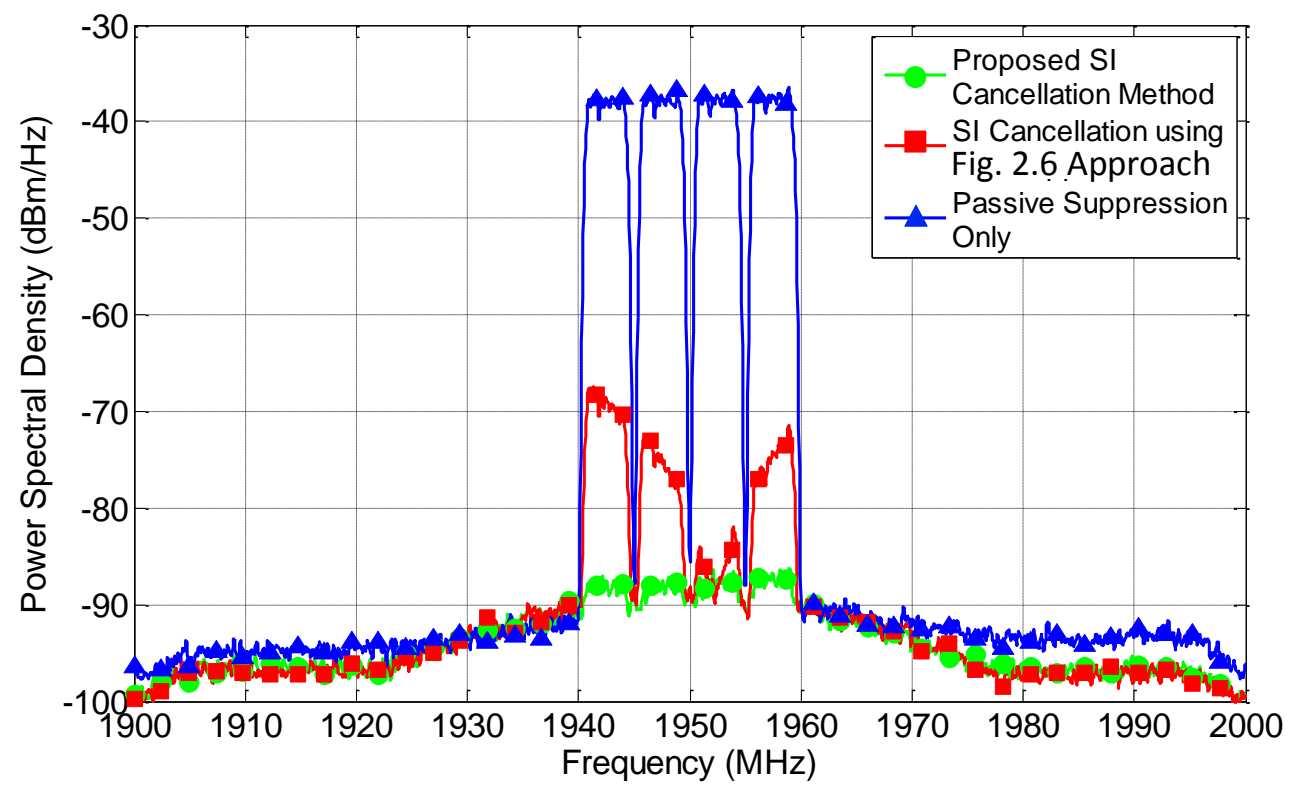

Figure 4.3: RF-Analog SI Cancellation of $20 \mathrm{MHz}$ Modulation Bandwidth Signal 


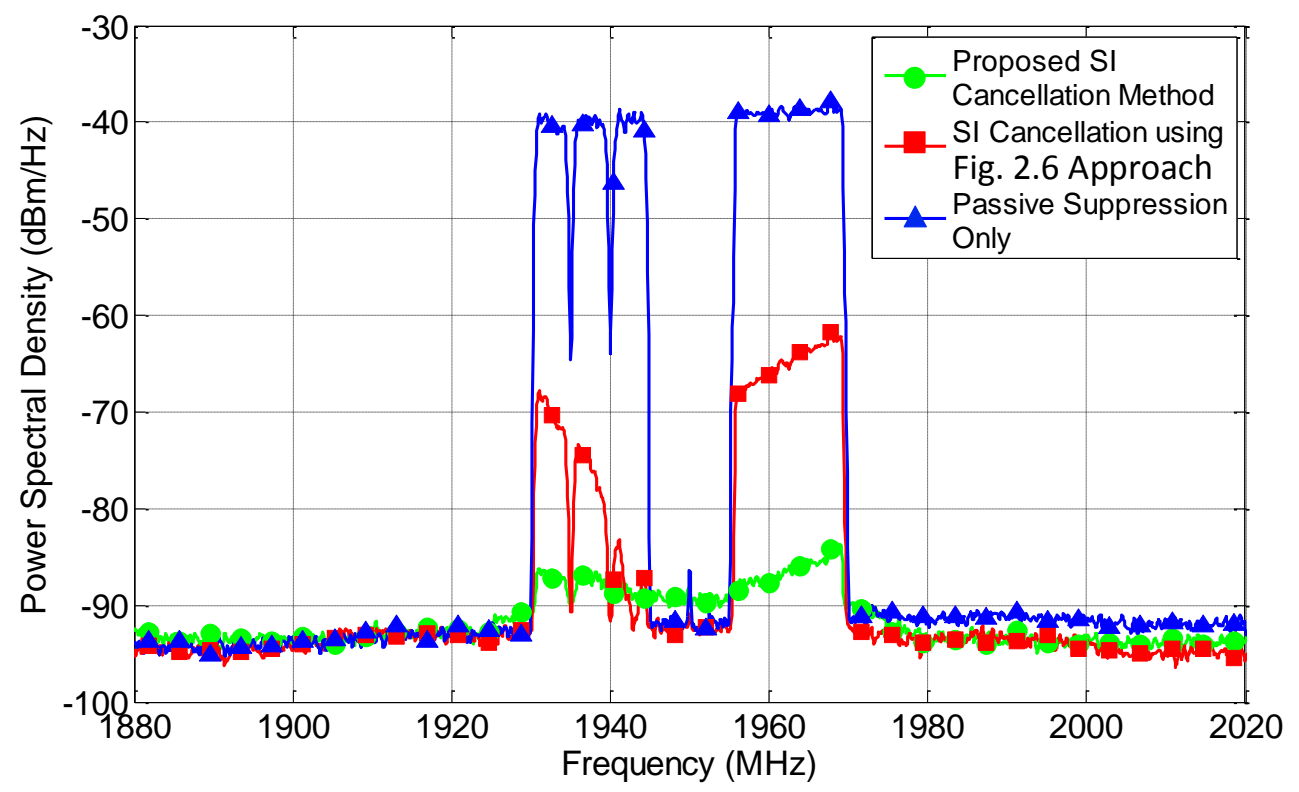

Figure 4.4: RF-Analog SI Cancellation of $40 \mathrm{MHz}$ Modulation Bandwidth Signal

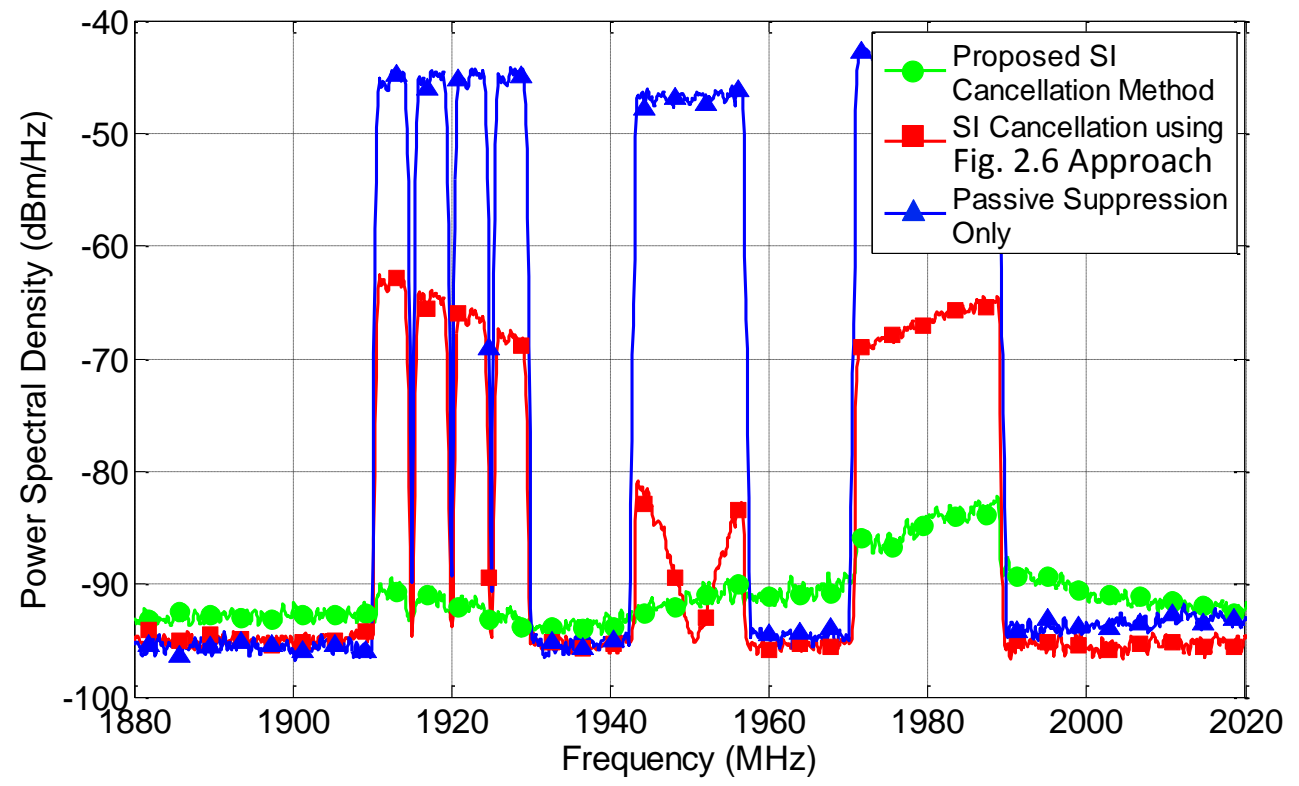

Figure 4.5: RF-Analog SI Cancellation of $80 \mathrm{MHz}$ Modulation Bandwidth Signal 


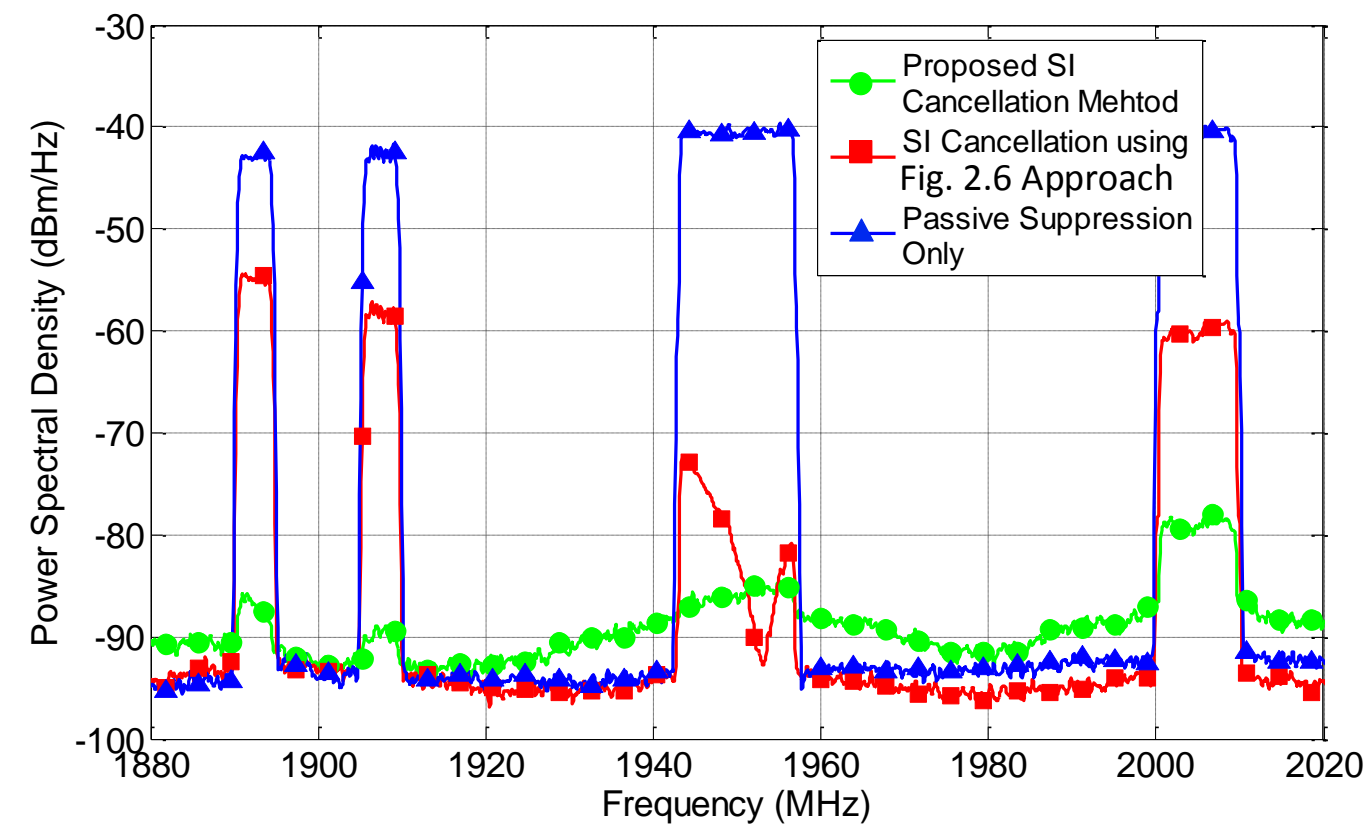

Figure 4.6: RF-Analog SI Cancellation of $120 \mathrm{MHz}$ Modulation Bandwidth Signal 


\subsection{System Analysis}

This section will review the previously discussed topics on nonidealities in the system for the proposed approach.

Although a significant amount of cancellation was achieved in the RF-analog domain, it is still not enough to entirely suppress the SI signal below the noise floor. Therefore, the receiver will have to tolerate the residual SI signal until it can be cancelled further through digital cancellation.

Analysis of the ADC quantization noise, discussed in Section 2.4.1, provides an approximation of how many bits will effectively be lost due to the SI signal. With $50 \mathrm{~dB}$ of RF-analog SI cancellation achieved through this topology for a $20 \mathrm{MHz} \mathrm{TX}$ signal and $23 \mathrm{~dB}$ of passive suppression, it follows that the power of the SI signal is around $-50 \mathrm{dBm}$. Therefore the only variable left in (2.2) is the amount of received signal power. The amount of bits lost versus the received signal power is plotted in Fig. 4.7.

The linearity of the LNA is discussed in Section 2.4.2, and results in a minimum required II $P_{3}$ value of the LNA to achieve third order intermodulation components below the noise floor, that depends on its maximum input power. The input power for this experimental setup includes the $-50 \mathrm{dBm}$ SI signal and the received signal power. The required $I I P_{3}$ value of the LNA versus the received signal power is also plotted in Fig. 4.7.

Fig. 4.7 establishes a system tradeoff where the quantization noise of the signal of interest in minimized when its signal power is increased, but this in turn increases the $I I P_{3}$ value of the LNA. A compromise is achieved when the signal of interest has a power level around $-60 \mathrm{dBm}$ of peak power, the number of bits lost is two and the required $I I P_{3}$ value is below $-25 \mathrm{dBm}$.

Phase noise in this topology is less of a concern because the TX signal is sampled after the PA so the canceller chain does not have an additional local oscillator. Therefore, the SI signal is decreased from RF-analog cancellation through the accuracy of the SI signal channel estimation.

The measurement results in Fig. 4.3, 4.4, 4.5, 4.6, show a slight degradation in cancellation with wider modulation bandwidth signals. In particular, the right side of Fig. 4.5 and Fig. 4.6 are degraded. This is most likely a result of the narrowband VM calibration that was done, the initial control signal that was used, and the resolution of the control signal. 


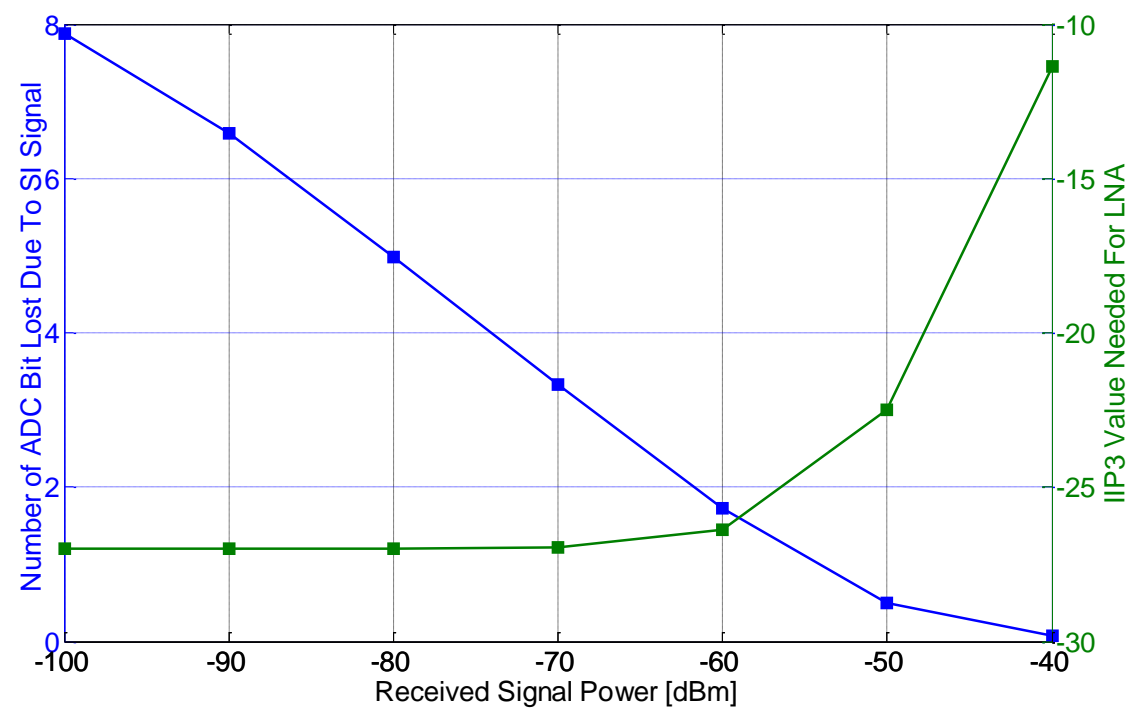

Figure 4.7: Number of ADC Bits Lost Due to the SI Signal and Require IIP3 Value of the LNA vs. the Received Signal Power

\subsection{Summary}

The proposed digitally-assisted RF-analog SI cancellation achieves excellent performance over the maximum modulation bandwidth that the equipment allowed. The results of the five TX test signals are summarized in Table 4.1.

Table 4.1: Summary of the Proposed SI Canceller Measurement Results

\begin{tabular}{cc}
\hline Modulation Bandwidth & Cancellation \\
\hline \hline CW & $55 \mathrm{~dB}$ \\
$20 \mathrm{MHz}$ & $50 \mathrm{~dB}$ \\
$40 \mathrm{MHz}$ & $47 \mathrm{~dB}$ \\
$80 \mathrm{MHz}$ & $42 \mathrm{~dB}$ \\
$120 \mathrm{MHz}$ & $40 \mathrm{~dB}$ \\
\hline
\end{tabular}

Many RF-analog SI cancellation techniques have been proposed recently in literature. Although some implementations include passive suppression and digital cancellation in 
their total cancellation reported, a summary of the cancellation from the RF-analog canceller alone is concluded in Table 4.2.

Table 4.2: Performance Comparisons of Other RF-Analog SI Cancellers

\begin{tabular}{cccc}
\hline Research Group & Design Description & Modulation Bandwidth & Cancellation \\
\hline \hline Stanford [3] & Balun inversion & $40 \mathrm{MHz}$ & $25 \mathrm{~dB}$ \\
Rice [9] & Auxiliary TX chain & $625 \mathrm{KHz}$ & $33 \mathrm{~dB}$ \\
Stanford [4] & $\begin{array}{c}\text { RF FIR filter with weighted } \\
\text { attenuators, 8 taps }\end{array}$ & $80 \mathrm{MHz}$ & $40 \mathrm{~dB}$ \\
Tampere [5] & VM Adjustment, 3 taps & $80 \mathrm{MHz}$ & $40 \mathrm{~dB}$ \\
Columbia [31] & Multiple RF bandpass filters & $20 \mathrm{MHz}$ & $20 \mathrm{~dB}$ \\
This Work & VM and Rational Function & $120 \mathrm{MHz}$ & $40 \mathrm{~dB}$ \\
& FIR Filter & \\
\hline
\end{tabular}




\section{Chapter 5}

\section{Conclusions}

System-level implementations for FD radios have been investigated in this thesis. The main issue encountered with the implementation of FD radios is the SI signal from TX power leaking into the RX chain. The analysis in Chapter 2 suggests that a large amount of SI cancellation before the RX chain is critical for a FD radio. This relaxes the bit requirements for the $\mathrm{ADC}$ or reduces the quantization noise and it improves the linearity of the RX chain so that the nonlinearities do not have to be compensated through digital cancellation. Sampling the TX signal after the PA also improves upon a lot of issues with FD radios as it embeds the TX chain nonidealities into the cancellation chain and it reduces the phase noise as there is no oscillator needed for the cancellation.

Review from the literature of RF-analog cancellers in Chapter 2 shows that the general magnitude and phase adjustment topology struggles to cancel wide modulation bandwidth signals. Instead, cancellation was widened through the implementation of an FIR filter in the RF-analog domain to mimic the isolation path's frequency dependency. However, additional nonidealties from the RF-analog components and complexity from these components make this topology unsuitable for practical implementations.

A new approach for RF-analog SI cancellation is presented in this thesis. This new topology builds upon the general magnitude and phase adjustment canceller and extends it to wide modulation bandwidth signals without increasing the number of taps in the RF-analog domain. The canceller is built using off-the-shelf components and experimental measurements using this initial prototype have demonstrated over $40 \mathrm{~dB}$ of SI cancellation can be achieved with a modulation bandwidth of up to $120 \mathrm{MHz}$. This bandwidth can also be extended with a wider bandwidth signal analyzer as well.

The advantage of this cancellation approach, beyond the good cancellation performance 
achieved, is its ability to cancel wide modulation bandwidths, its optimization of system nonidealities, and its hardware simplicity in the RF-analog domain. The identification of the digital filter parameters also only requires a single iteration compared to the multiple needed for other wideband solutions. The complexity that was seen previously in the RF-domain of other wideband RF-analog cancellers has been moved to the digital domain through the use of a rational function FIR filter.

\section{$5.1 \quad$ Future Works}

The aim of this thesis was to create a new RF-analog SI canceller that relaxed the complexity in the RF-analog domain and achieved cancellation over wide modulation bandwidths. In the current implementation, investigation into the exact limitations of the RF-analog canceller can be done. There residual SI signal degrades over wider modulation bandwidths and can possibly be improved further. However, this is just one piece of the FD radio in a static environment. Further development can be done on the digital cancellation portion, to obtain a complete FD radio. There are many techniques reported in the literature that would aid this system in achieving cancellation of the SI signal below the receiver noise floor. With this implementation, a practical receiver can also be included. As well, a perfect $50 \mathrm{ohm}$ termination was used at the antenna interface of the circulator instead of a real antenna for this initial prototype. Designing a simple antenna for the system would add antenna mismatch and reflections from the environment into the system. The RF-analog SI canceller can be expanded upon to adapt to these reflections to mimic a practical scenario. Furthermore, the canceller can easily be integrated to reduce the overall size of the system. Since the initial prototype was developed with off-the-shelf components, the size of the system is currently unrealistic for many applications.

It is also important that the canceller is tested on the current and upcoming trends

in communication networks. Specifically, higher frequencies of operation, cancellation of intermodulation from carrier aggregation, and for multi-input multi-output (MIMO) applications. 


\section{References}

[1] B. van Liempd, B. Debaillie, J. Craninckx, C. Lavin, C. Palacios, S. Malotaux, J. Long, D. van den Broek, and E. Klumperink, "Rf self-interference cancellation for full-duplex," in Cognitive Radio Oriented Wireless Networks and Communications (CROWNCOM), 2014 9th International Conference on, June 2014, pp. 526-531.

[2] J. I. Choi, M. Jain, K. Srinivasan, P. Levis, and S. Katti, "Achieving single channel, full duplex wireless communication," in Proceedings of the sixteenth annual international conference on Mobile computing and networking. ACM, 2010, pp. 1-12.

[3] M. Jain, J. I. Choi, T. Kim, D. Bharadia, S. Seth, K. Srinivasan, P. Levis, S. Katti, and P. Sinha, "Practical, real-time, full duplex wireless," in Proceedings of the 17th annual international conference on Mobile computing and networking. ACM, 2011, pp. 301-312.

[4] D. Bharadia, E. McMilin, and S. Katti, "Full duplex radios," in ACM SIGCOMM Computer Communication Review, vol. 43, no. 4. ACM, 2013, pp. 375-386.

[5] J. Tamminen, M. Turunen, D. Korpi, T. Huusari, Y.-S. Choi, S. Talwar, and M. Valkama, "Digitally-controlled rf self-interference canceller for full-duplex radios."

[6] A. Sabharwal, P. Schniter, D. Guo, D. Bliss, S. Rangarajan, and R. Wichman, "Inband full-duplex wireless: Challenges and opportunities," Selected Areas in Communications, IEEE Journal on, vol. 32, no. 9, pp. 1637-1652, Sept 2014.

[7] Y.-S. Choi and H. Shirani-Mehr, "Simultaneous transmission and reception: Algorithm, design and system level performance," Wireless Communications, IEEE Transactions on, vol. 12, no. 12, pp. 5992-6010, December 2013.

[8] D. Korpi, T. Riihonen, V. Syrjala, L. Anttila, M. Valkama, and R. Wichman, "Fullduplex transceiver system calculations: Analysis of adc and linearity challenges," 
Wireless Communications, IEEE Transactions on, vol. 13, no. 7, pp. 3821-3836, July 2014.

[9] M. Duarte and A. Sabharwal, "Full-duplex wireless communications using off-the-shelf radios: Feasibility and first results," in Signals, Systems and Computers (ASILOMAR), 2010 Conference Record of the Forty Fourth Asilomar Conference on, Nov 2010, pp. 1558-1562.

[10] M. Duarte, C. Dick, and A. Sabharwal, "Experiment-driven characterization of fullduplex wireless systems," Wireless Communications, IEEE Transactions on, vol. 11, no. 12, pp. 4296-4307, December 2012.

[11] E. Ahmed, A. Eltawil, and A. Sabharwal, "Rate gain region and design tradeoffs for full-duplex wireless communications," Wireless Communications, IEEE Transactions on, vol. 12, no. 7, pp. 3556-3565, July 2013.

[12] E. Everett, M. Duarte, C. Dick, and A. Sabharwal, "Empowering full-duplex wireless communication by exploiting directional diversity," in Signals, Systems and Computers (ASILOMAR), 2011 Conference Record of the Forty Fifth Asilomar Conference on, Nov 2011, pp. 2002-2006.

[13] K. Haneda, E. Kahra, S. Wyne, C. Icheln, and P. Vainikainen, "Measurement of loopback interference channels for outdoor-to-indoor full-duplex radio relays," in Proceedings of the Fourth European Conference on Antennas and Propagation, April 2010, pp. $1-5$.

[14] A. Khandani, "Methods for spatial multiplexing of wireless two-way channels," Oct. 19 2010, uS Patent 7,817,641.

[15] A. Sabharwal, P. Schniter, D. Guo, D. W. Bliss, S. Rangarajan, and R. Wichman, "Inband full-duplex wireless: Challenges and opportunities," IEEE Journal on Selected Areas in Communications, vol. 32, no. 9, pp. 1637-1652, 2014.

[16] B. Debaillie, D.-J. van den Broek, C. Lavin, B. van Liempd, E. Klumperink, C. Palacios, J. Craninckx, B. Nauta, and A. Parssinen, "Analog/rf solutions enabling compact full-duplex radios," Selected Areas in Communications, IEEE Journal on, vol. 32, no. 9, pp. 1662-1673, Sept 2014.

[17] E. Everett, A. Sahai, and A. Sabharwal, "Passive self-interference suppression for full-duplex infrastructure nodes," Wireless Communications, IEEE Transactions on, vol. 13, no. 2, pp. 680-694, February 2014. 
[18] L. Laughlin, M. A. Beach, K. A. Morris, and J. L. Haine, "Optimum single antenna full duplex using hybrid junctions," IEEE Journal on Selected Areas in Communications, vol. 32, no. 9, pp. 1653-1661, Sept 2014.

[19] B. van Liempd, B. Hershberg, K. Raczkowski, S. Ariumi, U. Karthaus, K.-F. Bink, and J. Craninckx, "2.2 a ¿70dbm iip3 single-ended electrical-balance duplexer in 0.18um soi cmos," in Solid- State Circuits Conference - (ISSCC), 2015 IEEE International, Feb 2015, pp. 1-3.

[20] D. Korpi, L. Anttila, V. Syrjala, and M. Valkama, "Widely linear digital selfinterference cancellation in direct-conversion full-duplex transceiver," Selected Areas in Communications, IEEE Journal on, vol. 32, no. 9, pp. 1674-1687, Sept 2014.

[21] L. Anttila, D. Korpi, V. Syrjala, and M. Valkama, "Cancellation of power amplifier induced nonlinear self-interference in full-duplex transceivers," in Signals, Systems and Computers, 2013 Asilomar Conference on, Nov 2013, pp. 1193-1198.

[22] E. Ahmed, A. Eltawil, and A. Sabharwal, "Self-interference cancellation with nonlinear distortion suppression for full-duplex systems," in Signals, Systems and Computers, 2013 Asilomar Conference on, Nov 2013, pp. 1199-1203.

[23] D. Korpi, T. Huusari, Y.-S. Choi, L. Anttila, S. Talwar, and M. Valkama, "Digital self-interference cancellation under nonideal rf components: Advanced algorithms and measured performance," in Signal Processing Advances in Wireless Communications (SPAWC), 2015 IEEE 16th International Workshop on, June 2015, pp. 286-290.

[24] A. Sahai, G. Patel, C. Dick, and A. Sabharwal, "On the impact of phase noise on active cancelation in wireless full-duplex," Vehicular Technology, IEEE Transactions on, vol. 62, no. 9, pp. 4494-4510, Nov 2013.

[25] E. Ahmed and A. M. Eltawil, "All-digital self-interference cancellation technique for full-duplex systems," IEEE Transactions on Wireless Communications, vol. 14, no. 7, pp. 3519-3532, July 2015.

[26] R. Askar, T. Kaiser, B. Schubert, T. Haustein, and W. Keusgen, "Active selfinterference cancellation mechanism for full-duplex wireless transceivers," in Cognitive Radio Oriented Wireless Networks and Communications (CROWNCOM), 2014 9th International Conference on, June 2014, pp. 539-544.

[27] T. Huusari, Y. S. Choi, P. Liikkanen, D. Korpi, S. Talwar, and M. Valkama, "Wideband self-adaptive rf cancellation circuit for full-duplex radio: Operating principle and 
measurements," in 2015 IEEE 81st Vehicular Technology Conference (VTC Spring), May 2015, pp. 1-7.

[28] J. G. McMichael and K. E. Kolodziej, "Optimal tuning of analog self-interference cancellers for full-duplex wireless communication," in Communication, Control, and Computing (Allerton), 2012 50th Annual Allerton Conference on, Oct 2012, pp. 246251.

[29] J. Zhou, T.-H. Chuang, T. Dinc, and H. Krishnaswamy, "19.1 receiver with ¿20mhz bandwidth self-interference cancellation suitable for fdd, co-existence and full-duplex applications," in Solid- State Circuits Conference - (ISSCC), 2015 IEEE International, Feb 2015, pp. 1-3.

[30] J. Zhou and H. Krishnaswamy, "Recent developments in fully-integrated rf selfinterference cancellation for frequency-division and full-duplex radios," in Vehicular Technology Conference (VTC Spring), 2015 IEEE 81st, May 2015, pp. 1-5.

[31] J. Zhou, T.-H. Chuang, T. Dinc, and H. Krishnaswamy, "Integrated wideband selfinterference cancellation in the rf domain for fdd and full-duplex wireless," Solid-State Circuits, IEEE Journal of, vol. 50, no. 12, pp. 3015-3031, Dec 2015.

[32] Q. Gu, RF system design of transceivers for wireless communications. Springer Science \& Business Media, 2005.

[33] B. Razavi, RF microelectronics. Prentice Hall New Jersey, 1998, vol. 1.

[34] T. Dinc, A. Chakrabarti, and H. Krishnaswamy, "A 60 ghz cmos full-duplex transceiver and link with polarization-based antenna and rf cancellation," IEEE Journal of Solid-State Circuits, vol. 51, no. 5, pp. 1125-1140, May 2016.

[35] L. Anttila, D. Korpi, V. Syrjl, and M. Valkama, "Cancellation of power amplifier induced nonlinear self-interference in full-duplex transceivers," in 2013 Asilomar Conference on Signals, Systems and Computers, Nov 2013, pp. 1193-1198.

[36] D. Korpi, L. Anttila, and M. Valkama, "Feasibility of in-band full-duplex radio transceivers with imperfect rf components: Analysis and enhanced cancellation algorithms," in Cognitive Radio Oriented Wireless Networks and Communications (CROWNCOM), 2014 9th International Conference on, June 2014, pp. 532-538.

[37] A. Sahai, G. Patel, C. Dick, and A. Sabharwal, "Understanding the impact of phase noise on active cancellation in wireless full-duplex," in Signals, Systems and Computers 
(ASILOMAR), 2012 Conference Record of the Forty Sixth Asilomar Conference on, Nov 2012, pp. 29-33.

[38] V. Syrjala, M. Valkama, L. Anttila, T. Riihonen, and D. Korpi, "Analysis of oscillator phase-noise effects on self-interference cancellation in full-duplex ofdm radio transceivers," Wireless Communications, IEEE Transactions on, vol. 13, no. 6, pp. 2977-2990, June 2014.

[39] D. J. van den Broek, E. A. M. Klumperink, and B. Nauta, "A self-interference cancelling front-end for in-band full-duplex wireless and its phase noise performance," in Radio Frequency Integrated Circuits Symposium (RFIC), 2015 IEEE, May 2015, pp. $75-78$.

[40] A. Oppenheim and R. Schafer, Digital Signal Processing, ser. Prentice Hall international editions. Prentice-Hall, 1975.

[41] "Agilent fundamentals of arbitrary waveform generation: A high performance awg primer," M8190-91050, 2012.

[42] E. Nash, "Correcting imperfections in iq modulators to improve rf signal fidelity," AN-1039, Application Note, Analog Devices, 2009.

[43] J. Xia, E. Ng, and S. Boumaiza, "Wide-band compensation of rf vector multiplier for rf predistortion systems," IEEE Transactions on Circuits and Systems II: Express Briefs, vol. PP, no. 99, pp. 1-1, 2016. 